\title{
Thermodynamic Theory of Diffusion and Thermodiffusion Coefficients in
} Multicomponent Mixtures

\section{Shapiro, Alexander A.}

Published in:

Journal of Non-Equilibrium Thermodynamics

Link to article, DOI:

10.1515/jnet-2020-0006

Publication date:

2020

Document Version

Publisher's PDF, also known as Version of record

Link back to DTU Orbit

Citation (APA):

Shapiro, A. A. (2020). Thermodynamic Theory of Diffusion and Thermodiffusion Coefficients in Multicomponent Mixtures. Journal of Non-Equilibrium Thermodynamics, 45(4), 343-372. https://doi.org/10.1515/jnet-2020-0006

\section{General rights}

Copyright and moral rights for the publications made accessible in the public portal are retained by the authors and/or other copyright owners and it is a condition of accessing publications that users recognise and abide by the legal requirements associated with these rights.

- Users may download and print one copy of any publication from the public portal for the purpose of private study or research.

- You may not further distribute the material or use it for any profit-making activity or commercial gain

- You may freely distribute the URL identifying the publication in the public portal

If you believe that this document breaches copyright please contact us providing details, and we will remove access to the work immediately and investigate your claim 


\title{
Research Article
}

\section{Alexander A. Shapiro* \\ Thermodynamic Theory of Diffusion and Thermodiffusion Coefficients in Multicomponent Mixtures}

https://doi.org/10.1515/jnet-2020-0006

Received January 27, 2020; revised May 10, 2020; accepted June 25, 2020

\begin{abstract}
Transport coefficients (like diffusion and thermodiffusion) are the key parameters to be studied in non-equilibrium thermodynamics. For practical applications, it is important to predict them based on the thermodynamic parameters of a mixture under study: pressure, temperature, composition, and thermodynamic functions, like enthalpies or chemical potentials. The current study develops a thermodynamic framework for such prediction. The theory is based on a system of physically interpretable postulates; in this respect, it is better grounded theoretically than the previously suggested models for diffusion and thermodiffusion coefficients. In fact, it translates onto the thermodynamic language of the previously developed model for the transport properties based on the statistical fluctuation theory. Many statements of the previously developed model are simplified and amplified, and the derivation is made transparent and ready for further applications. The $n(n+1) / 2$ independent Onsager coefficients are reduced to $2 n+1$ determining parameters: the emission functions and the penetration lengths. The transport coefficients are expressed in terms of these parameters. These expressions are much simplified based on the Onsager symmetry property for the phenomenological coefficients. The model is verified by comparison with the known expressions for the diffusion coefficients that were previously considered in the literature.
\end{abstract}

Keywords: transport coefficients, diffusion, thermodiffusion, mixture, thermodynamic theory

\section{Introduction}

The transport coefficients in multicomponent mixtures (diffusion, thermodiffusion, heat conductivity, viscosity) are of major importance for the different areas of engineering and natural science. For practical applications, it is important to have model expressions making it possible to evaluate these coefficients based on the thermodynamic properties of a mixture. Modeling is essential, since experimental data are scarce and difficult to obtain, and only a few experimental values are available for mixtures containing more than two components [1,2]. The transport coefficients may also be accessed by molecular dynamics simulations, which are outside of the scope of the present work (see, e. g., the reviews in [3-5]).

The diffusion coefficients are often obtained by application of the Darken [6], Vignes [7], or more advanced mixing rules $[8,9]$ to the coefficients in the dilute solution limits. The last coefficients are calculated by the famous Einstein-Stokes formula, or the Wilke-Chang equation, or their modifications [10]. The mixing rules may be applied to both Fickian and Maxwell-Stefan diffusion coefficients [11]. This approach may only predict monotonous dependencies of the diffusion coefficients on molar fractions, which does not always correspond to the experimental data [12-14] (the thesis [14] contains also a comprehensive review of the different approaches to the diffusivity modeling). Other approaches involve the group contribution or local composition models [15-18]; the models based on the Eyring absolute reaction rate concepts (activation energy, free volume) [19-22]; and direct or indirect correlation with viscosity [23, 24]. The recently proposed "swapping"

*Corresponding author: Alexander A. Shapiro, CERE-Center for Energy Resources Engineering, Department of Chemical and Biochemical Engineering, Technical University of Denmark, DTU b. 229, 2800 Kgs. Lyngby, Denmark, e-mail: ash@kt.dtu.dk 
model should also be mentioned [25]. All these models are essentially empirical or semi-empirical. The fully theoretically grounded expressions for diffusion coefficients exist only for the dilute gases: for the Knudsen flow [26] and for the flow governed by the kinetic Boltzmann equation [26, 27], although the problems with the Onsager symmetry may arise in higher approximations [28, 29].

Thermodynamic models for the thermodiffusion coefficients are usually based on the concept of the heats of transport introduced by Denbigh [30, 31]. In the models, these values are identified with the different thermodynamic quantities, like specific enthalpies or energies, or their combinations with some correction factors [32-41] (see also reviews [42-44]). For diluted gas mixtures, the answers, again, are given by the gas kinetic theory [45]. Recently, a simplified approach to thermodiffusion was suggested specifically for the transport in porous media [46]. The simplification is needed since the experimental determination of the thermodiffusion coefficients in multicomponent mixtures is a difficult task [47, 48].

In summary, the thermodynamic models for diffusion and thermodiffusion coefficients are built independently of each other, and they are based on the different physical principles. Non-equilibrium thermodynamics is a unifying theory behind the transport processes in the mixtures. This theory, in its general form, proves, on the basis of the statistical thermodynamics of fluctuations, the symmetry limitations to the transport coefficients, but it does not specify their values and their dependencies on the equilibrium thermodynamic properties [32, 49, 50].

We previously derived expressions for prediction of the transport properties, based on the statistical thermodynamics and the fluctuation theory [51,52]. The matrix of Onsager coefficients was evaluated on the basis of the traditional thermodynamic variables and the newly introduced quantities, the so-called penetration lengths. The new expressions were successfully applied to the evaluation of the diffusion coefficients in simple and complex mixtures [12-14]. Moreover, it was possible to evaluate the penetration lengths independently, by molecular dynamics simulations [53]. Modeling the thermodiffusion coefficients was less accurate [42]. The model required a penetration length for energy; compared with the penetration lengths for the molecules, this value was more loosely defined, and could not be approximated by any simple dependence. Despite the fundamental background, the developed theory had a number of flaws. The derivations were long and physically non-transparent. The fundamental property of symmetry of the Onsager transport coefficients did not automatically follow from the derivation and had to be imposed post-factum.

The present work proposes a unified thermodynamic theory for the diffusion and thermodiffusion coefficients (though not applicable to other transport properties, like viscosities). Our goal is not to calculate specific coefficients, but to provide a framework for thermodynamic modeling. The approach is based upon but does not fully repeat the assumptions and derivations of the works [51, 52]. Unlike those works, we have tried to set out the theory on the macroscopic language of thermodynamics, with a minimum reference to the molecular statistical mechanics. The new description appears to be much simpler conceptually and is based now on a number of physically meaningful postulates. The Onsager property of symmetry of the phenomenological coefficients is one of these postulates.

We consider a classical discontinuous two-vessel system connected by a conducting element [26,30]. The mass and energy balances are obeyed in the system, though the force balance is non-trivial due to interactions between the fluid and the walls. The system is close to equilibrium, so that the fluxes in it approach the fluxes under fluctuation, as required by the Onsager symmetry derivation. It is postulated that for such flows the number of molecules emitted from a vessel to the conductor per unit time depends only on the thermodynamic state of the vessel. This value, called the emission function, serves as a potential for the molecular flow. Not all the molecules penetrate via the conductor; their fraction reaching the opposite vessel is called the penetration probability (for the continuous systems, it is transformed to the penetration length). The sets of emission functions and penetration lengths determine the molecular flow. Similar functions are introduced for energy flow. The Onsager symmetry relations impose strong limitations on the possible choice of emission and penetration functions. Thus, the set of independent phenomenological coefficients is reduced to the two sets of simpler functions. The number of new functions grows linearly with the number of components, unlike the number of Onsager coefficients growing quadratically. These functions may be obtained from the model considerations or molecular dynamics simulations. We demonstrate how the diffusion and thermodiffusion 


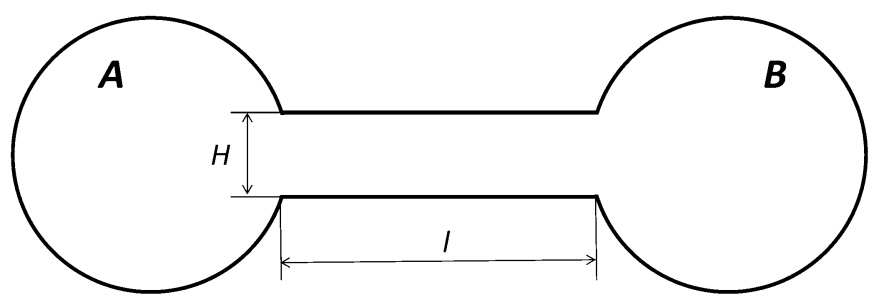

Figure 1: Scheme of a two-vessel system.

coefficients are expressed in terms of the emission and penetration functions, and how the known results about diffusion and thermodiffusion may be recovered in the new modeling scheme.

The paper is organized as follows. The next subsection introduces basic designations and background theoretical concepts of the paper. Section 2 discusses the theory of the flow of the multicomponent Knudsen gas. This theory is well studied, and all the transport coefficients for the dilute Knudsen gas are known. The goal of its re-discussion here is to introduce the emission functions and penetration probabilities in a natural way, which later will be generalized onto arbitrary multicomponent mixtures. This generalization is carried out in Section 3. We introduce the governing dependencies for the arbitrary multicomponent flow and derive restrictions on the transport coefficients imposed by the Onsager reciprocal relations. The transition from the discontinuous to continuous systems (from effusion to diffusion) is also discussed in Sections 2 and 3. Section 4 compares the results of the developed theoretical framework with the known formulae for diffusion coefficients in binary mixtures. The thermodiffusion coefficients are also discussed.

\subsection{Background knowledge and agreements about designations}

In this work, we consider a system of two vessels $A$ and $B$ connected by a pipe, or a tube, or a capillary conducting the flow (Figure 1). For brevity, we will call this element "a conductor." This is a traditional "discontinuous" system for the study of the flows in non-equilibrium thermodynamics [26, 31]. It serves as a natural generalization of a typical one-vessel system (the PVT cell), which is considered in equilibrium thermodynamics. Otherwise, it has all the limitations of a near-equilibrium discontinuous system, like those listed above. The transfer of the results regarding diffusion and thermodiffusion coefficients from the discontinuous to the continuous description will be discussed

Any characteristics $Z$ of the fluid in vessel $A(B)$ will be denoted as $Z_{A}\left(Z_{B}\right)$, while its average value will be denoted, simply, by $Z$. We assume that the differences $\Delta Z=Z_{B}-Z_{A}$ are small and the second-order corrections with regard to $\Delta Z$ may be neglected. Each vessel is in equilibrium, but these equilibria may be different for vessels $A$ and $B$. The amount of substance in the conductor is small compared with the vessels. We consider a model two-vessel system, but not a real one, and the walls of the conductor are assumed to be fully reflective. The force balance between the mixture and the walls of the system and, therefore, the analysis of stresses in the mixture are not considered. A detailed analysis of the flow interacting with the walls is outside the scope of the present work (see, e. g., [54, 55]).

The system is saturated by an ordinary mixture of several components. The thermodynamic state is characterized by pressure $P$, temperature $T$, and composition. The composition will be expressed in terms of mass fractions $c_{i}$ or individual component mass densities $\rho_{i}(i=1, \ldots, n)$. The molar fractions $z_{i}$ will also be used. The components in the mixture are assumed to be non-reacting and non-adsorbing on the walls of the system. Action of external fields, like gravity, is neglected.

An object of study is the component and energy fluxes in the conductor created by the differences of thermodynamic parameters between the vessels. For an $n$-component mixture, there are $n$ mass fluxes and one energy flux $J_{i}(i=1, \ldots, n+1)$. The designations $J_{i}$ are meant to be used for fluxes per cross-sectional area $H$ of the conductor, so that the total flows are equal to $H J_{i}$. 
The entropy production in the system has the form of

$$
\frac{d S}{d t}=H \sum_{i=1}^{n+1} J_{i} F_{i}
$$

Here $F_{i}$ are thermodynamic forces conjugate to the thermodynamic fluxes $J_{i}$. Normally, these forces have the form of $\zeta_{i} \Delta Z_{i}$, for some thermodynamic variables $Z_{i}$, or linear combinations of such values.

The Onsager reciprocal relations read

$$
J_{i}=\sum_{j=1}^{n+1} L_{i j} F_{j}(i=1, \ldots, n+1) .
$$

The matrix of phenomenological coefficients $\boldsymbol{L}=\left[L_{i j}\right]$ was proven to be symmetric for the "even" processes in simple molecular systems, like diffusion and thermodiffusion $[56,57]$ :

$$
L_{i j}=L_{j i}(i, j=1, \ldots, n+1) .
$$

We exclude the motion under the action of the magnetic field, where some coefficients may be antisymmetric [49].

The goal of the present paper is to express coefficients $L_{i j}$ in terms of the physically well-defined values.

\section{Knudsen gas revisited}

In this section, we revisit the classical theory of transport of the Knudsen gas in the discontinuous system described above. A Knudsen gas is defined as a dilute gas mixture of non-interacting molecules. The Onsager theory for a single-component monatomic gas was described by Waldmann [54] (see also [58]). Our goal is not to re-discover long known dependencies and formulae, but, using the Knudsen gas as a fully studied example, to formulate such an approach to transport that may be applicable to more advanced systems. Simultaneously, we demonstrate and resolve some difficulties arising under such an extension.

\subsection{A single-component gas: The role of the symmetry condition}

Initially, the differences of pressure $P$ and temperature $T$ are created between vessels $A$ and $B$, causing substance (mass) and energy flows through the conductor. The molecules in the conductor do not interact, so that all of them that leave vessel $A$ reach vessel $B$, and vice versa. The energy flow between the vessels is solely due to the molecular flow.

The general laws for such flows may be represented in the form of

$$
\begin{gathered}
J_{m}=-\Delta \Gamma, \\
J_{E}=-\Delta \mathrm{E} .
\end{gathered}
$$

Here $\Gamma, E$ are the amounts of substance and energy emitted for each vessel per unit of time. We will call $\Gamma$, E the emission functions, or the emission expressions. Later we will generalize these expressions onto more complex cases.

For the Knudsen gas,

$$
\Gamma=\frac{1}{4} \rho C, C=\left(\frac{8 R T}{\pi M}\right)^{\frac{1}{2}},
$$

where $\rho$ is density and $C$ is the average molecular velocity. The multiplier $\frac{1}{4}$ reflects the fact that not all the molecules are directed towards another vessel, and that only a projection of their velocity onto the conductor direction $x$ contributes to the flow. 
A classical monatomic gas is the gas with the heat capacities $c_{V}=\frac{3}{2} R$ and $c_{P}=\frac{5}{2} R$. Waldmann [54] postulates the energy emission for such gas $E$ to be $\frac{1}{4} C \cdot 2 P$. While the multiplier $\frac{1}{4} C$ is of the same nature as for the mass transfer (energy of the Knudsen gas is only transferred with the flowing molecules), the multiplier $2 P$ is less trivial. Waldmann shows that with such a value of $E$ the transport coefficients obey the Onsager symmetry relations. Moreover, it is easy to demonstrate that this is the only possible value of $E$ retaining the symmetry. However, the physical interpretation of the energy emission $2 P$ still remains unclear.

We will repeat computations for a polyatomic gas and, more generally, for a fluid with arbitrary thermodynamic dependencies $\rho(P, T), \mathrm{E}(P, T)$. First, we express eqs. (1) and (2) in terms of the pressure and temperature differences:

$$
\begin{aligned}
& J_{m}=-\frac{C}{4}\left(\rho_{P} \Delta P+\left(\rho_{T}+\frac{\rho}{2 T}\right) \Delta T\right) \\
& J_{E}=-\left(\mathrm{E}_{P} \Delta P+\mathrm{E}_{T} \Delta T\right) .
\end{aligned}
$$

The heat flux $J_{Q}$ is now defined as

$$
J_{Q}=J_{E}-h J_{m}=-\frac{1}{4}\left[\left(4 \mathrm{E}_{P}-h C \rho_{P}\right) \Delta P+\left(4 \mathrm{E}_{T}-h C \rho_{T}-\frac{h C \rho}{2 T}\right) \Delta T\right],
$$

where $h$ is the enthalpy per unit mass. The entropy production in the system is [54]

$$
\frac{d S}{d t}=H\left[\left(-\frac{\Delta P}{\rho T}\right) J_{m}+\left(-\frac{\Delta T}{T^{2}}\right) J_{Q}\right]
$$

Note that the entropy production should be multiplied by cross-section $H$ in order to convert fluxes per area to total fluxes.

Hence, the fluxes $J_{m}, J_{Q}$ are conjugate to forces $-\frac{\Delta P}{\rho T},-\frac{\Delta T}{T^{2}}$, respectively:

$$
\left[\begin{array}{c}
J_{m} \\
J_{Q}
\end{array}\right]=\left[\begin{array}{ll}
L_{m m} & L_{m Q} \\
L_{Q m} & L_{Q Q}
\end{array}\right]\left[\begin{array}{c}
-\frac{\Delta P}{\rho T} \\
-\frac{\Delta T}{T^{2}}
\end{array}\right]
$$

Combining eqs. (4)-(7), we obtain

$$
\left[\begin{array}{ll}
L_{m m} & L_{m Q} \\
L_{Q m} & L_{Q Q}
\end{array}\right]=\frac{T}{4}\left[\begin{array}{cc}
\rho \rho_{P} & C T\left(\rho_{T}+\frac{\rho}{2 T}\right) \\
\rho\left(4 \mathrm{E}_{P}-h C \rho_{P}\right) & T\left(4 \mathrm{E}_{T}-h C \rho_{T}-\frac{h C \rho}{2 T}\right)
\end{array}\right] .
$$

This matrix is symmetric if the cross-diagonal elements are equal:

$$
\mathrm{E}_{P}=\frac{1}{4} C\left(h \rho_{P}+T \frac{\rho_{T}}{\rho}+\frac{1}{2}\right)
$$

For the monatomic ideal gas $\rho=M P / R T$ and $h=5 R T / 2 M$. Then the last expression gives exactly $\mathrm{E}=$ $\frac{1}{4} C \cdot 2 P$. For the classical polyatomic gas, with constant heat capacities and $h=c_{P} R T / M$, eq. (8) results in

$$
\mathrm{E}=\frac{1}{4 M} C \cdot\left(c_{P}-\frac{R}{2}\right) \rho T=\frac{1}{4 M} C \cdot\left(c_{V}+\frac{R}{2}\right) \rho T .
$$

The first addendum in this expression, $C c_{V} \rho T / 4 M$, is due to energy transfer with the molecules, since $c_{V} T$ is the energy per mole and $C \rho / 4 M$ is the molecular flow ( $N=\rho / M$ is the number of moles). However, the question remains about the appearance of the second addendum, $C \rho / 4 M \cdot R T / 2$ in the last expression. Its meaning is not transparent, and the expression without it, from the first glance, seems to be more appropriate.

An explanation based on gas kinetic theory is given in Appendix A. The reason is, roughly, that the fast molecules are emitted more often and carry more kinetic energy than the slow molecules. The addendum proportional to $R T / 2$ appears from the difference $\left\langle v_{x} \cdot \frac{m v^{2}}{2}\right\rangle-\left\langle v_{x}\right\rangle\left\langle\frac{m v^{2}}{2}\right\rangle$, where the average is taken over the velocity vectors with a positive component $v_{x}$. This example shows that the emission expressions may be rather non-trivial. More examples are provided below. 


\subsection{Generalization}

\subsubsection{Gas mixture}

The theory from Section 2.1 may be generalized into a multicomponent ideal gas mixture. This generalization is not fully trivial since the entropy involves now the entropy of mixing. For an $n$-component mixture of noninteracting species eq. (1) is substituted by $n$ equations

$$
J_{m, i}=-\Delta \Gamma_{i}(i=1, \ldots, n),
$$

while the energy equation becomes

$$
J_{E}=-\Delta \sum_{k=1}^{n} \mathrm{E}_{k}
$$

The entropy production assumes the form [49]

$$
\frac{d S}{d t}=H \sum_{i=1}^{n} \Delta\left(-\frac{\mu_{i}}{T}\right) J_{m, i}+H \Delta\left(\frac{1}{T}\right) J_{E} .
$$

Here $\mu_{i}$ are chemical potentials per unit mass of a component. The mass-based system of variables is more convenient for the flow problems than the mole-based system, which is more usual in equilibrium thermodynamics. A reason is that in hydrodynamics a momentum balance equation is necessarily formulated in terms of mass densities. The necessary thermodynamic formula in the mass system of variables is presented in Appendix B. It is convenient to introduce designations

$$
v_{i}=-\frac{\mu_{i}}{T} ; \tau=\frac{1}{T} .
$$

Equation (12) indicates that $\Delta v_{i}, \Delta \tau$ are thermodynamic force conjugates, respectively, to the thermodynamic fluxes $J_{m, i}, J_{E}$. In order to determine the matrix of Onsager coefficients, eqs. (10) and (11) are expanded with regard to $\Delta v_{i}, \Delta \tau$ :

$$
\begin{gathered}
J_{m, i}=-\sum_{j=1}^{n} \frac{\partial \Gamma_{i}}{\partial v_{j}} \Delta v_{j}-\frac{\partial \Gamma_{i}}{\partial \tau} \Delta \tau(i=1, \ldots, n) ; \\
J_{E}=-\sum_{k=1}^{n} \sum_{j=1}^{n} \frac{\partial \mathrm{E}_{k}}{\partial v_{j}} \Delta v_{j}-\sum_{k=1}^{n} \frac{\partial \mathrm{E}_{k}}{\partial \tau} \Delta \tau .
\end{gathered}
$$

It follows from the last two equations that the phenomenological coefficients for this system are

$$
\begin{gathered}
L_{i j}=-\frac{\partial \Gamma_{i}}{\partial v_{j}}(i, j=1, \ldots n) ; \\
L_{i, n+1}=-\frac{\partial \Gamma_{i}}{\partial \tau} ; L_{n+1, i}=-\sum_{k=1}^{n} \frac{\partial \mathrm{E}_{k}}{\partial v_{i}}(i=1, \ldots, n) ; \\
L_{n+1, n+1}=-\sum_{k=1}^{n} \frac{\partial \mathrm{E}_{k}}{\partial \tau} .
\end{gathered}
$$

The symmetry conditions are, therefore,

$$
\begin{gathered}
\frac{\partial \Gamma_{i}}{\partial v_{j}}=\frac{\partial \Gamma_{j}}{\partial v_{i}} \\
\sum_{k=1}^{n} \frac{\partial \mathrm{E}_{k}}{\partial v_{i}}=\frac{\partial \Gamma_{i}}{\partial \tau}
\end{gathered}
$$


Assume, as above in eq. (3), that the mass emissions for a multicomponent Knudsen gas have the form of

$$
\Gamma_{i}=\frac{1}{4} \rho_{i} C_{i}, C_{i}=\left(\frac{8 R T}{\pi M_{i}}\right)^{\frac{1}{2}}
$$

Then, as follows from eq. (62) in Appendix B, condition (17) is obeyed automatically, since $\rho_{i}$ depend only on $T$ and $v_{i}$ (with the same number $i$ ), and matrix $\left\{\partial \Gamma_{i} / \partial v_{j}\right\}$ is diagonal. Condition (18) assumes the form [see eq. (63)]

$$
\frac{\partial}{\partial v_{i}} \sum_{k=1}^{n} \mathrm{E}_{k}=-\frac{C_{i} \rho_{i}\left(c_{V, i}+\frac{R}{2}\right)}{R \tau} .
$$

Substitution of $\rho_{i}$ from eq. (61) and integration over $v_{i}$ results in

$$
\sum_{k=1}^{n} \mathrm{E}_{k}=\sum_{k=1}^{n} \frac{C_{i} \rho_{i}\left(c_{V, i}+\frac{R}{2}\right)}{M_{i} \tau}+A_{0}(T)
$$

The arbitrary constant of integration $A_{0}(T)$ may be eliminated, since the energy emission should be zero if all densities $\rho_{i}$ are zeros. Equation (20) should, in principle, be sufficient for the determination of the total energy emission $\sum_{k=1}^{n} \mathrm{E}_{k}$, since only the total emission is required to be known in eq. (11). If, additionally, individual values $\mathrm{E}_{i}$ have to be found, it may be assumed that each $\mathrm{E}_{i}$ depends on the partial density $\rho_{i}$, but not on the densities of other components. This assumption is reasonable since the components of the ideal gas mixture are independent and non-interacting. Then, finally,

$$
\mathrm{E}_{i}=\frac{C_{i} \rho_{i}\left(c_{V, i}+\frac{R}{2}\right)}{M_{i} \tau} .
$$

This derivation shows that the energy and the mass emissions are connected to each other. A connection is given by the Onsager reciprocal relations. The energy emission may be computed on the basis of the mass emissions.

\subsubsection{Penetration probabilities and penetration lengths}

The flow equations for a single-component gas (1) and (2) and for a multicomponent gas (10) and (11) are based on an inherent assumption that all the molecules that are emitted from vessel $A$ reach vessel $B$ and vice versa. The conductor conducts all the molecules; no molecule is reflected back and returns to the original vessel, and no molecule loses or gains energy when interacting with the conductor.

This assumption is not self-evident. It may be true if the conductor is straight and has mirror-reflecting walls, where the $x$-component of the molecular velocity and its internal energy remain unchanged in a collision, while the transversal component of the molecular momentum changes its sign. However, most of the surfaces reflect molecules in a diffuse way, or they are close to that (the accommodation coefficients are close to unity) [59]. Even in a straight conductor, some molecules may be reflected and return to the original vessel that they left. The situation with the energy is even less definite.

If a conductor is tortuous, the probability of back-reflection increases. The theory is often applied to porous membranes or other media consisting of such capillaries. In order to be able to generalize the considerations to these types of conductors, we introduce the molecular penetration probabilities $p_{i}$ and the energy penetration probabilities $p_{E, i}$. We assume that a probability of penetration from $A$ to $B$ is equal to that from $B$ to $A$. Otherwise, the conductor would support different equilibrium compositions in the vessels and, thus, be capable of the mixture separation, which is outside the scope of the present work. The corrected eqs. (10) 
and (11) acquire the form

$$
\begin{gathered}
J_{m, i}=-p_{i} \Delta \Gamma_{i}(i=1, \ldots, n) ; \\
J_{E}=-\sum_{k=1}^{n} p_{E, k} \Delta \mathrm{E}_{k} .
\end{gathered}
$$

Further computations of the previous subsection are only slightly modified. The expressions for phenomenological coefficients (14) to (16) become

$$
\begin{gathered}
L_{i j}=-p_{i} \frac{\partial \Gamma_{i}}{\partial v_{j}}(i, j=1, \ldots n) ; \\
L_{i, n+1}=-p_{i} \frac{\partial \Gamma_{i}}{\partial \tau} ; L_{n+1, i}=-\sum_{k=1}^{n} p_{E, i} \frac{\partial \mathrm{E}_{k}}{\partial v_{i}}(i=1, \ldots, n) ; \\
L_{n+1, n+1}=-\sum_{k=1}^{n} p_{E, i} \frac{\partial \mathrm{E}_{k}}{\partial \tau} .
\end{gathered}
$$

For an ideal gas, the coefficients (24) remain diagonal, and the Onsager symmetry condition holds for them. The symmetry conditions for coefficients $L_{i, n+1}$ and $L_{n+1, i}$ look more sophisticated. We may assume again that $\mathrm{E}_{i}$ depend only on $\rho_{i}$ or $v_{i}$ (with the same $i$ ), since in an ideal gas mixture, emissions of the different components should be independent. Then condition (21) is transformed into

$$
\mathrm{E}_{i}=-\int \frac{p_{i}}{p_{E, i}} \frac{C_{i} \rho_{i}\left(c_{V, i}+\frac{R}{2}\right)}{R \tau} d v_{i}
$$

Simplification and integration in eq. (27) are possible if the ratio $p_{i} / p_{E, i}$ is constant or only temperaturedependent. This condition is reduced to eq. (21) if $p_{i}=p_{E, i}$ (that is, energy is transferred together with the molecules). More generally, it is reduced to

$$
\mathrm{E}_{i}=\frac{p_{i}}{p_{E, i}} \frac{C_{i} \rho_{i}\left(c_{V, i}+\frac{R}{2}\right)}{M_{i} \tau} .
$$

The multiplier $p_{i} / p_{E, i}$ is a correction to the energy emission necessary to establish the correspondence between the energy transfer with the substance and the substance transfer due to the energy flow (Soret and Dufour effects). It may be discussed whether this symmetry is necessarily valid for the Knudsen gas since the fundamental Onsager derivation of the symmetry property presumes a certain degree of molecular interaction. The goal of this work, however, is to generalize the theory to systems of the interacting molecules, where symmetry of Onsager coefficients is verified by experimental data [60]. If the limiting transition from interacting to non-interacting systems is, in a way, smooth, then the symmetry should be preserved.

Another problem to be discussed here is the transition between discontinuous and continuous systems. While in the discontinuous two-vessel systems the fluxes are proportional to the differences $\Delta \Gamma_{i}, \Delta \mathrm{E}_{i}$, in the continuous systems, they should be proportional to the spatial derivatives or gradients of these values. Transition to the derivatives is formally performed by division on the length $l$ of the conductor so that the differences like $\Delta \Gamma / l$ are substituted by the derivatives $\partial \Gamma / \partial x$. We introduce the penetration distances $\lambda_{i}, \lambda_{E, i}$, defined as

$$
\lambda_{i}=p_{i} l ; \lambda_{E, i}=p_{E, i} l
$$

Physical meanings of these values will be discussed further, in Section 3.3 and Appendix D, for a more general case. The continuous transport equations corresponding to the discontinuous eqs. (22) and (23) are

$$
\begin{gathered}
J_{m, i}=-\lambda_{i} \frac{\partial \Gamma_{i}}{\partial x}(i=1, \ldots, n) ; \\
J_{E}=-\sum_{k=1}^{n} \lambda_{E, k} \frac{\partial \mathrm{E}_{k}}{\partial x} .
\end{gathered}
$$




\subsubsection{Diffusion, heat, and convective fluxes}

Equations (29) and (30) have the form of Fourier and Fick equations for the corresponding fluxes. A difference is, however, that eq. (29) is formulated for the total mass fluxes. Meanwhile, the Fick law is related to the diffusion fluxes, which are defined as deviations of the individual component fluxes from the average convective flux. Similarly, the Fourier law is related to the non-convective heat flux, but not the total energy flux. A reason is that the momentum equation is commonly formulated in terms of the convective flux; hence, it should be separated from other fluxes. Transition to the diffusion and heat fluxes is considered in this subsection.

The convective, diffusion, and heat fluxes are defined as [14, 32, 61]

$$
J_{c}=\sum_{i=1}^{n} J_{m, i} ; J_{D, i}=J_{m, i}-c_{i} J_{c}(i=1, \ldots, n) ; J_{Q}=J_{E}-h J_{c} .
$$

Here $c_{i}$ are mass fractions of the different components. Out of the diffusion fluxes, only $n-1$ are independent, since their sum is equal to zero. We select $J_{D, 1}, \ldots, J_{D, n-1}$ as such fluxes.

It is convenient to make vectors (rows) with the fluxes as elements. We introduce vectors $\boldsymbol{J}_{M}=\left(J_{m, 1}, \ldots\right.$, $\left.J_{m, n}, J_{E}\right)^{T}$ and $\boldsymbol{J}_{D}=\left(J_{D, 1}, \ldots, J_{D, n-1}, J_{Q}, J_{C}\right)^{T}$. Both vectors have a dimension of $n+1$. As follows from eq. (31), they are connected by the matrix relation

$$
\boldsymbol{J}_{D}=\boldsymbol{K}_{D M} \boldsymbol{J}_{M} ; \boldsymbol{K}_{D M}=\left(\begin{array}{cccccc}
1-c_{1} & -c_{1} & \cdots & -c_{1} & -c_{1} & 0 \\
-c_{2} & 1-c_{2} & \cdots & -c_{2} & -c_{2} & 0 \\
\vdots & \vdots & \ddots & \vdots & \vdots & \vdots \\
-c_{n-1} & -c_{n-1} & \cdots & 1-c_{n-1} & -c_{n-1} & 0 \\
-h & -h & \cdots & -h & -h & 1 \\
1 & 1 & \cdots & 1 & 1 & 0
\end{array}\right) .
$$

The systems of thermodynamic fluxes correspond to the systems of thermodynamic forces $\boldsymbol{F}_{M}, \boldsymbol{F}_{D}$. The correspondence is established via the following expression for the entropy production:

$$
\frac{d S}{d t}=H \boldsymbol{J}_{M}^{T} \boldsymbol{F}_{M}=H \boldsymbol{J}_{D}^{T} \boldsymbol{F}_{D}
$$

As follows from eqs. (12) and (13), the vector of thermodynamic forces corresponding to $\boldsymbol{J}_{M}$ is $\boldsymbol{F}_{M}=$ $\left(\Delta v_{1}, \ldots, \Delta v_{M}, \Delta \tau\right)^{T}$. It is derived in Appendix $C$ that the thermodynamic forces corresponding to $\boldsymbol{J}_{D}$ are

$$
\boldsymbol{F}_{D}=\left(\Delta\left(v_{1}-v_{n}\right), \ldots, \Delta\left(v_{n-1}-v_{n}\right), \Delta \tau,-\frac{\tau}{\rho} \Delta P\right)^{T} .
$$

That is, the thermodynamic force corresponding to the heat flux is the temperature difference, and the convective flux is conjugate to the normalized pressure difference, as expected.

The matrices of phenomenological Onsager coefficients relate thermodynamic fluxes and forces:

$$
\boldsymbol{J}_{M}=\boldsymbol{L}_{M} \boldsymbol{F}_{M}, \boldsymbol{J}_{D}=\boldsymbol{L}_{D} \boldsymbol{F}_{D}
$$

Coefficients in matrix $\boldsymbol{L}_{M}$ are given by eqs. (24), (26) of the previous subsection. Matrix $\boldsymbol{L}_{D}$ is obtained from $\boldsymbol{L}_{M}$ by the following transformation preserving the symmetry $[49,50]$ :

$$
\boldsymbol{L}_{D}=\boldsymbol{K}_{D M} \boldsymbol{L}_{M} \boldsymbol{K}_{D M}^{T}
$$

For the coefficients of the matrix $\boldsymbol{L}_{M}$ given by expressions (24)-(26), expressions for the coefficients of the matrix $\boldsymbol{L}_{D}$ become rather cumbersome. We will present the explicit expressions for the case of a binary 
mixture:

$$
\begin{gathered}
L_{D, 11}=-c_{2}^{2} \lambda_{1} \frac{\partial \Gamma_{1}}{\partial v_{1}}-c_{1}^{2} \lambda_{2} \frac{\partial \Gamma_{2}}{\partial v_{2}} \\
L_{D, 22}=-h \lambda_{1}\left(h \frac{\partial \Gamma_{1}}{\partial v_{1}}-\frac{\partial \Gamma_{1}}{\partial \tau}\right)-h \lambda_{2}\left(h \frac{\partial \Gamma_{2}}{\partial v_{2}}-\frac{\partial \Gamma_{2}}{\partial \tau}\right)-\sum_{i=1}^{2} \lambda_{E, i} \frac{\partial E_{i}}{\partial \tau} ; \\
L_{D, 33}=-\lambda_{1} \frac{\partial \Gamma_{1}}{\partial v_{1}}-\lambda_{2} \frac{\partial \Gamma_{2}}{\partial v_{2}} ; \\
L_{D, 21}=L_{D, 12}=h\left(c_{2} \lambda_{1} \frac{\partial \Gamma_{1}}{\partial v_{1}}-c_{1} \lambda_{2} \frac{\partial \Gamma_{2}}{\partial v_{2}}\right)-c_{2} \lambda_{1} \frac{\partial \Gamma_{1}}{\partial \tau}+c_{1} \lambda_{2} \frac{\partial \Gamma_{2}}{\partial \tau} ; \\
L_{D, 31}=L_{D, 13}=-c_{2} \lambda_{1} \frac{\partial \Gamma_{1}}{\partial v_{1}}+c_{1} \lambda_{2} \frac{\partial \Gamma_{2}}{\partial v_{2}} ; \\
L_{D, 32}=L_{D, 23}=-h\left(\lambda_{1} \frac{\partial \Gamma_{1}}{\partial v_{1}}+\lambda_{2} \frac{\partial \Gamma_{2}}{\partial v_{2}}\right)+\lambda_{1} \frac{\partial \Gamma_{1}}{\partial \tau}+\lambda_{2} \frac{\partial \Gamma_{2}}{\partial \tau}
\end{gathered}
$$

In these expressions, we have substituted the penetration probabilities $p_{i}$ with the penetration distances $\lambda_{i}$, as discussed in the previous subsection. We have also applied the symmetry of the Onsager coefficients, excluding, as much as possible, the terms where the energy emission function is present. As a result, the energy emission appears only in the heat conductivity coefficient $L_{D, 22}$. All the other coefficients may be calculated if the mass emission functions and the molecular penetration distances are known.

More precisely, the roles of the different coefficients are as follows. The diagonal coefficients $L_{D, 11}, L_{D, 22}$, $L_{D, 33}$ are conductivities for diffusion, heat, and convective fluxes with regard to the driving forces $\Delta\left(v_{1}-v_{2}\right)$, $\Delta \tau,-\left(\frac{\tau}{\rho}\right) \Delta P$, respectively. The off-diagonal coefficients express coupling between these fluxes. In particular, coefficients $L_{D, 12}=L_{D, 21}$ express coupling between heat conduction and diffusion (Soret and Dufour effects). Other off-diagonal coefficients express coupling with convection: of diffusion (coefficients $L_{D, 13}=L_{D, 31}$ ) and of heat conduction (coefficients $L_{D, 23}=L_{D, 32}$ ).

Coupling between diffusive and convective fluxes, as well as between heat conduction and convection, is an unexpected property worth discussing. In continuous systems, the corresponding fluxes are not coupled. The convective fluxes stay separately from diffusion and heat conduction. They are independent of thermodynamic variables or their gradients. A complete discussion of the dependent and independent thermodynamic forces and fluxes for such systems is presented in [62]. Their transport coefficients (viscosities) are not directly related to diffusion or heat conductivity coefficients, unless in some specific models. However, diffusion and heat conduction are coupled. A physical background behind the uncoupling of the convective flow from other fluxes is that it is driven by the viscous forces, having a different tensor dimension than other thermodynamic forces. They are independent according to the Curie principle. Also, the Galilean invariance shows that the relative diffusion velocities must be independent of the convective velocity.

In discontinuous systems, like the system considered here, there is a fixed system of coordinates related to the conductor, and the Galilean invariance does not hold. All the fluxes, including the convective flux, are conditioned by the thermodynamic states of the vessels. If the molecules interact with each other and with the walls, then a viscous structure of the flow is formed (like the Poiseuille flow in straight cylindrical capillaries). Nevertheless, the convective and diffusive/heat flows should not be coupled. This was proven, e. g., in [63] for a more general case of flows in macroporous media ("a conductor consisting of a great many capillaries"). Although the basic flow equation for flows in porous media (the Darcy law) involves the same tensor dimensions as equations for diffusion and heat flows, it may still be proven that these equations must be independent.

The Knudsen gas considered here is a gas of non-interacting molecules. Its dynamic viscosity is not defined, being "a product of zero by infinity" (density by molecular free path). It takes infinite time for the Knudsen gas to establish a viscous flow structure in the conductor. Therefore, coupling between diffusion and convection, as well as between heat flow and convection, is still possible. In Section 3.4, we will show that in discontinuous systems, there may be coupling between diffusion and convection even for denser fluids if the viscous structure of the flow is not established or postulated. 
It should be noted, finally, that a tedious exercise of separation of diffusive and convective fluxes is unnecessary for the Knudsen gas since simple and straightforward description based on eqs. (22) and (23) [or (29) and (30)] is more advantageous. Here it has been carried out in order to demonstrate coupling and other arising problems. The derivation is rather general, and later will be generalized to dense mixtures.

\subsection{Conclusion}

The goal of the present section has been to formulate the theory of Knudsen gas flow in such a way that it may be extended into more complex systems. The theory of Knudsen gas flow in a discontinuous two-vessel system may be derived based on the simple eqs. (1) and (2) [or, in the multicomponent case, from eqs. (10) and (11)]. Key functions for this extension are the emission functions $\Gamma_{i}, E_{i}$ and the penetration probabilities $p_{i}, p_{E, i}$ (or the penetration lengths $\lambda_{i}, \lambda_{E, i}$ ).

The energy transfer-related parameters $\mathrm{E}_{i}, p_{E, i}$ are needed to determine the heat conductivity coefficient, while all the other coefficients need only knowledge of the mass transfer-related functions $\Gamma_{i}, p_{i}$. The "trick" is to utilize Onsager symmetry relations in order to exclude the energy transfer from the off-diagonal coefficients of the Onsager matrix. Then the transition from discontinuous to continuous systems may be carried out, simply, by substituting the penetration probabilities with the penetration lengths. If the transition from the mass and energy fluxes to the diffusion, convection, and heat fluxes is needed, then the transformations described in Section 2.2.3 may be applied. The problem of coupling between the fluxes, which are commonly treated as uncoupled for the continuous systems, has been discussed.

Some derivations of this section might be regarded as unnecessary equivalent transformations since the emission functions for the Knudsen gas are known in advance, while the penetration probabilities are solely determined by the geometry of the conductor and properties of its walls. However, these derivations become a necessary part of the theory describing arbitrary mixtures, as demonstrated in the next section.

\section{General theory}

\subsection{Basic assumptions and axioms}

In this section, we will generalize the previously described Knudsen gas theory onto arbitrary non-ideal mixtures. We will utilize the axiomatic approach, formulating several relatively simple and transparent statements, from which the rest of the theory will be derived.

As in the previous section, we consider a discontinuous system consisting of the two vessels connected by the conductor. The molecules are emitted from the vessels, while their transfer happens in the conductor. The total amount of substance transferred from vessel $A$ to vessel $B$ consists of the molecules emitted from $A$, apart from those which got reflected in the conductor and returned. The same is valid for transfer from $B$ to $A$.

It is convenient to split the process into emission and conduction. The numbers of molecules emitted from the vessels per unit time depend only on what happens inside the vessels, but not in the conductor. Since the vessels are in equilibrium, these numbers are equilibrium functions of the thermodynamic states of the vessels.

Assumption 1. Consider, for definiteness, vessel $A$. Define values $\Gamma_{i}$, being masses of species $i$ emitted from the vessel per unit time. It is assumed that $\Gamma_{i}$ depend only on the thermodynamic state of the vessel: pressure $P_{A}$, temperature $T_{A}$, and partial component densities $\rho_{A, i}$. The same, of course, is valid for vessel $B$.

It should be stressed that this assumption is only valid for the cases where the vessels may be considered to be in equilibrium and the conductor does not have a back influence on them. The vessels should be large enough compared with the conductor volume; and, on the other hand, the conductor should be long enough 
in order to separate the vessels from mutual influence. The system should be very close to equilibrium, so that the corrections related to the kinetic energy of the directed flow and diffusion fluxes should be of the second order of magnitude [62]. These statements are often assumed in the background for some experimental methods of diffusion measurements [14].

As stated above, only part of the molecules emitted from vessel $A$ reach $B$ and vice versa. The fraction $p_{i}$ of the molecules leaving $A$ and reaching $B$ will be called the penetration probability. The molecules may now be not only reflected from the conductor walls but also from other molecules. Hence, the penetration probability depends on the properties of the conductor, as well as the properties of the mixture in it. The mixture in the conductor is in a non-equilibrium state, so that, in principle, the probabilities $p_{i}$ may depend on the gradients of thermodynamic variables. We assume, however, that the considered deviations from equilibrium are small, so that dependence of $p_{i}$ on the gradients may be neglected.

For any thermodynamic property $Z$, the average between the values $Z_{A}$ and $Z_{B}$ will simply be denoted by $Z$. Then we formulate the following.

Assumption 2. The mass penetration probabilities $p_{i}$ (as well as energy penetration probabilities introduced below) depend on the average thermodynamic properties between the two vessels: $P, T, \rho_{i}$.

Finally, we assume that the conductor cannot separate the mixtures.

Assumption 3. In the equilibrium state all the values $P, T, \rho_{i}$ are equal for vessels $A$ and $B$.

These three assumptions are sufficient to formulate the mass transfer equations in the form (22). In particular, assumption excludes the situation where the penetration probabilities are unequal for molecules moving from $A$ to $B$ and from $B$ to $A$. This may be demonstrated in the following way. Assume temporarily that the penetration probabilities in the different directions are not equal; denote them by $p_{A B, i}$ and $p_{B A, i}$. Instead of eq. (22), we will obtain an equation

$$
J_{m, i}=p_{A B, i} \Gamma_{A, i}-p_{B A, i} \Gamma_{B, i} .
$$

In equilibrium the fluxes $J_{m, i}$ should be zero, which is only possible if $\Gamma_{A, i} \neq \Gamma_{B, i}$. Therefore, the thermodynamic characteristics of the vessels must also be unequal, in contradiction to Assumption 3.

While the mass transfer equations for dense gases and liquids may still be expressed in the form (22), the equations for energy transfer must be modified. The reason is that, apart from convective energy transfer with the moving molecules, there may also be non-convective transfer, where the energy is moving through the system by passing from one molecule to another via molecular interactions.

The following scheme for energy emission may look excessive and cumbersome from the first glance; however, this is probably the minimum possible description suitable for both rarefied Knudsen gas and dense liquid. It will be shown that, despite the abundance of the introduced functions, all the transport coefficients may be defined in terms of mass emission functions and penetration probabilities, apart from the heat conductivity coefficients. The symmetry of the Onsager matrix makes it possible to do so. Moreover, some strong limitations for the energy emission functions will also be derived.

We assume that the energy emission function may be divided into two parts: the energy traveling with the emitted molecules and the energy $\mathrm{E}_{X, n c}$ propagating in a non-convective way, by interaction of the molecules in the vessel and molecules emitted from it and the molecules in the conductor, without the mass exchange. Then we have

$$
\mathrm{E}_{X}=\sum_{i=1}^{n} \Gamma_{X, i} D_{X, i}+\mathrm{E}_{X, n c}(X=A, B)
$$

When a molecule from vessel $A$ (for example) appears in the conductor, its carried energy $D_{A, i}$ is divided. Part of this energy $G_{A, i}$ keeps the value acquired in the vessel (corresponding to the thermodynamic conditions in it). Another part $g_{i}$ exchanges energy with other molecules of the conductor. The same is, of course, valid for the molecules emitted from vessel $B$. Within the approximation of small differences between the vessels, 
it may be assumed that $g_{i}$ corresponds to the average thermodynamic characteristics in the conductor, that is, the average between $A$ and $B$ :

$$
D_{X, i}=G_{X, i}+g_{i}(X=A, B) .
$$

The energy flux is also divided into the convective and non-convective flux, similarly to the energy emission function. The non-convective conduction of energy is governed by the energy penetration probability $p_{E}$ :

$$
J_{E, n c}=-p_{E} \Delta \mathrm{E}_{n c} .
$$

The convective energy flux consists of the fluxes of preserved energy values $G_{i}$ and non-preserved values $g_{i}$. Since these values are moving with the molecules, the mass penetration probabilities must be involved:

$$
J_{E, c}=-\sum_{i=1}^{n} p_{i} \Delta\left(\Gamma_{i} G_{i}\right)-\sum_{i=1}^{n} p_{i} g_{i} \Delta \Gamma_{i} .
$$

The total flux is, simply, the sum of the convective and non-convective energy fluxes:

$$
J_{E}=-\sum_{i=1}^{n} p_{i} \Delta\left(\Gamma_{i} G_{i}\right)-\sum_{i=1}^{n} p_{i} g_{i} \Delta \Gamma_{i}-p_{E} \Delta \mathrm{E}_{n c} .
$$

In the Knudsen limit, only the first addendum remains, and the flux converges to the Knudsen expression with $p_{E, i}=p_{i}$. On the contrary, for the dense fluids, the first addendum disappears, since a molecule in the conductor rapidly acquires the average energy of the surrounding molecules. Formally,

$$
\begin{gathered}
\text { if } P \rightarrow 0 \text {, then } \mu_{i} \rightarrow-\infty, v_{i} \rightarrow \infty \text {, and } g_{i} \rightarrow 0, \mathrm{E}_{n c} \rightarrow 0 \text {; } \\
\text { if } P \rightarrow \infty \text {, then } \mu_{i} \rightarrow \infty, v_{i} \rightarrow-\infty \text {, and } G_{i} \rightarrow 0 .
\end{gathered}
$$

Assumption 4. The energy flux is described by eq. (40), with the functions obeying the limiting relations (41) and (42) (with the explanations given above).

The final assumption is related to the fact that the conductors may be different. They may have different tortuosities, be built from different materials, and, generally, have different selectivities to various molecules and energies. Each change of the conductor will affect the penetration probabilities, but not the emission functions. We assume that the variety of conductors is such that any imaginable combination of penetration probabilities (within obvious restrictions) can be realized without modification of the emission functions.

Assumption 5. For the different discontinuous systems, penetration probabilities $p_{i}, p_{E}$ in eqs. (22) and (40) may vary arbitrarily, independently of each other and of the emission functions.

\subsection{Onsager symmetry and its consequences}

As previously, we consider the system of thermodynamic "coordinates" consisting of variables $v_{i}=-\mu_{i} / T$ and $\tau=1 / T$. Derivation of expressions for Onsager coefficients is fully similar to Sections 2.2.1 and 2.2.2, with the only difference that the energy transport eq. (40) is more sophisticated. The resulting expressions for the Onsager coefficients are

$$
\begin{gathered}
L_{i j}=-p_{i} \frac{\partial \Gamma_{i}}{\partial v_{j}}(i, j=1, \ldots n) ; \\
L_{i, n+1}=-p_{i} \frac{\partial \Gamma_{i}}{\partial \tau} ; L_{n+1, i}=-\sum_{k=1}^{n} p_{k} \frac{\partial \Gamma_{k} G_{k}}{\partial v_{i}}-\sum_{k=1}^{n} p_{k} g_{k} \frac{\partial \Gamma_{k}}{\partial v_{i}}-p_{E} \frac{\partial \mathrm{E}_{n c}}{\partial v_{i}}(i=1, \ldots, n) ; \\
L_{n+1, n+1}=-\sum_{k=1}^{n} p_{k} \frac{\partial \Gamma_{k} G_{k}}{\partial \tau}-\sum_{k=1}^{n} p_{k} g_{k} \frac{\partial \Gamma_{k}}{\partial \tau}-p_{E} \frac{\partial \mathrm{E}_{n c}}{\partial \tau} .
\end{gathered}
$$


These expressions may be greatly simplified by the application of the Onsager symmetry relations. As stated in the Introduction, we assume these relations to be universally valid. The symmetry condition for the coefficients $L_{i j}(i, j=1, \ldots, n)$ is obtained on the basis of eq. (43):

$$
p_{i} \frac{\partial \Gamma_{i}}{\partial v_{j}}=p_{j} \frac{\partial \Gamma_{j}}{\partial v_{i}}
$$

According to Assumption 5, probabilities $p_{i}$ may vary independently of $\Gamma_{i}$. Hence, eq. (46) is only possible if the matrix of coefficients $\partial \Gamma_{i} / \partial v_{j}$ is diagonal. In other words, the following statement is proven.

Statement 1. The values of $\Gamma_{i}$ are independent of $v_{j}$ for $j \neq i$, i. e.,

$$
\Gamma_{i}=\Gamma_{i}\left(v_{i}, \tau\right)=\Gamma_{i}\left(\mu_{i}, T\right)
$$

This result may be expected based on the meaning of the chemical potential as the amount of energy needed to insert a molecule of species $i$ into the mixture. Emission is inverse to insertion, and that is why it is correlated to the individual insertion energy of the species.

Equality of the coefficients $L_{i, n+1}$ and $L_{n+1, i}$, accounting for Statement 1, results in an identity:

$$
p_{i} \frac{\partial \Gamma_{i}}{\partial \tau}=\sum_{k=1}^{n} p_{k} \Gamma_{k} \frac{\partial G_{k}}{\partial v_{i}}+p_{i}\left(g_{i}+G_{i}\right) \frac{\partial \Gamma_{i}}{\partial v_{i}}+p_{E} \frac{\partial \mathrm{E}_{n c}}{\partial v_{i}} .
$$

In the analysis of this expression, let us first vary $p_{E}$ independently of the mass penetration probabilities $p_{k}$. This is possible, according to Assumption 5. Identity (47) will be retained only if all $\partial \mathrm{E}_{n c} / \partial v_{i}$ are equal to zero. Hence, we have the following statement.

Statement 2. The value of non-convective energy transfer $\mathrm{E}_{n c}$ depends only on temperature $T$.

Now, due to Statement 2, the term $p_{E} \partial \mathrm{E}_{n c} / \partial v_{i}$ is removed from eq. (47). In the remaining equation, the probabilities $p_{k}$ may vary independently. Hence, the terms corresponding to the different $p_{l}$ should be equal independently of each other. Leaving only the terms containing $p_{i}$, we obtain

$$
\frac{\partial \Gamma_{i}}{\partial \tau}=\Gamma_{i} \frac{\partial G_{i}}{\partial v_{i}}+\left(g_{i}+G_{i}\right) \frac{\partial \Gamma_{i}}{\partial v_{i}}=\frac{\partial \Gamma_{i} G_{i}}{\partial v_{i}}+g_{i} \frac{\partial \Gamma_{i}}{\partial v_{i}} .
$$

If, additionally, we select the terms with $k \neq i$ in eq. (47), then we obtain

$$
\Gamma_{k} \frac{\partial G_{k}}{\partial v_{i}}=0
$$

From the last two equalities, the following statement follows.

Statement 3. Both $G_{i}$ and $g_{i}$ depend only on $v_{i}$ and $\tau$ (or $\mu_{i}$ and $T$ ).

Application of the three statements above results in a strong simplification of the kinetic coefficients. Equations (43) and (44) are reduced to

$$
\begin{gathered}
L_{i j}=-p_{i} \delta_{i j} \frac{\partial \Gamma_{i}\left(v_{i}, \tau\right)}{\partial v_{i}}(i, j=1, \ldots n) ; \\
L_{i, n+1}=-p_{i} \frac{\partial \Gamma_{i}}{\partial \tau} ; L_{n+1, i}=-p_{i} \frac{\partial \Gamma_{i} G_{i}}{\partial \nu_{i}}-p_{i} g_{i} \frac{\partial \Gamma_{i}}{\partial v_{i}}(i=1, \ldots, n) ;
\end{gathered}
$$

eq. (45) remains unchanged, but all the functions $\Gamma_{i}, G_{i}, g_{i}, E_{n c}$ entering this equation are also greatly simplified.

The expressions for Onsager coefficients $L_{i j}(i, j=1, \ldots n)$ and $L_{i, n+1}$ are identical to the expressions for the Knudsen gas, apart from unknown functions $\Gamma_{i}$. Moreover, coefficients $L_{n+1, i}$ may be substituted by $L_{i, n+1}$. The only difference with the Knudsen gas coefficients is in the expression for $L_{n+1, n+1}$. It is possible to compute all 
the coefficients except for the heat conductivity coefficient based on the two sets of functions, $\Gamma_{i}$ and $p_{i}$. This shows that the main assumptions of the proposed theory are rather restrictive.

Equation (48) provides additional information about the energy emission functions. For the dilute gas (but not necessarily Knudsen), the values of $g_{i}$ become zero, and eq. (48) becomes fully similar to eq. (18) for the Knudsen gas. In this limit, the values $\mathrm{E}_{k}$ become equal to $\Gamma_{k} G_{k}$, and it should be recalled that these values are only dependent on $v_{k}, \tau$.

In the opposite limit of a dense liquid or gas, the values of $G_{k}$ become equal to zero. In this limit eq. (48) is reduced to

$$
\frac{\partial \Gamma_{i}}{\partial \tau}=g_{i} \frac{\partial \Gamma_{i}}{\partial v_{i}}
$$

From this equation, the value of $g_{i}$ may be expressed and substituted into eq. (45) for the heat conductivity coefficient:

$$
L_{n+1, n+1}=-\sum_{k=1}^{n} p_{k}\left(\frac{\partial \Gamma_{k}}{\partial \tau}\right)^{2} / \frac{\partial \Gamma_{k}}{\partial v_{k}}-p_{E} \frac{\partial \mathrm{E}_{n c}}{\partial \tau} .
$$

Thus, in the limit of dense liquid the heat conductivity coefficients are determined by the mass emission functions, as well as values $p_{E}, \mathrm{E}_{n c}(\tau)$. Most of the energy emission functions are, again, excluded.

\subsection{Penetration probabilities and penetration lengths}

According to Section 2.2.2, a transition from penetration probabilities to penetration lengths is necessary in order to transfer from discontinuous to continuous systems. The probabilities are changed to the lengths as far as the differences of emission functions in the transport equations are substituted by their derivatives. The relationship between probabilities and lengths is described by eq. (28) (in particular, $\lambda_{i}=p_{i} l$ ). In this section, we discuss the physical meaning of the penetration lengths in more detail.

The introduction of the values $\lambda_{i}$ may look problematic, since it involves the length of the conductor $l$. From the first glance, the transport equations for continuous flows in free space should be free of any memory about the characteristics of a conductor. Otherwise, different values of diffusion and other coefficients would not only depend on the characteristics of the molecular motion, but also on geometry and other properties of a vessel or a pipe where this motion occurs.

This problem does not appear for the Knudsen gas considered in Section 2.2.2. Since the molecules in this gas do not interact, the molecular resistance to motion is zero, and the molecules move freely as long as the geometry of the conductor allows them to do so. Thus, the size and the geometry of the conductor determine the motion. A characteristic size of the conductor $l$ should enter transport coefficients. For example, the transport equations for the Knudsen gas in a porous medium contain characteristic pore sizes or equivalent parameters [26].

The situation becomes different for dense gases or liquids. In this case, interaction with other molecules becomes more significant than the limitations imposed by geometry of the conductor. The values of $\lambda_{i}$ should become, in principle, independent of this geometry. At least, this should be valid for "not-so-complex" conductors with reflecting walls. For such ideal conductors, penetration distances become absolute values depending on the molecular structure of the fluid, while penetration probabilities must, in principle, depend on the conductor length.

Independence of the penetration distances of the properties of a conductor may be explained in the following way. Consider a molecule of species $i$ emitted to the conductor, for example, from vessel $A$. At the moment of emission, an $x$-component of the molecule velocity will be positive and, on average, different from the velocities of the surrounding molecules in the conductor. Such a molecule will advance forward, towards the vessel $B$, until it will "forget" its initial velocity in the interactions with other molecules. After that moment it will participate in unbiased random walks, where displacements towards vessel $A$ or $B$ will be equiprobable. Denote by $\lambda_{i}$ an average distance that a molecule travels before forgetting its initial veloc- 
ity. A known random-walk theorem states that a probability $p_{i}$ that the molecule will reach vessel $B$ before returning to $A$ is equal to $\lambda_{i} / l$. This is exactly eq. (28).

Stochastic arguments showing that $p_{i}$ are given by the equality $\lambda_{i} / l$ are presented in Appendix D.

The considerations of this section rely upon the concept of "ideal conductor," which also needs a discussion. Consider the Knudsen number $K n=\lambda_{f} / l$, where $\lambda_{f}$ is a free molecular path between collisions. For the Knudsen gas, the value of $\lambda_{f}$ is efficiently equal to infinity, which makes the geometry of the conductor a determining factor. Meanwhile, if $\lambda_{f}$ is finite, it is always possible to select a conductor, whose size $l$, as well as thickness $H$, will be much larger than $\lambda_{f}$. It may be expected that the values of $\lambda_{i}$ will also be much smaller than $l$ (although different from $\lambda_{f}$ ). Most molecules will penetrate liquid or gas saturating the conductor colliding with other molecules much more often than with the conductor walls. So, the definition of the values of $\lambda_{i}$ as distances during which a molecule "forgets" its initial velocity becomes (almost) independent of the geometry of a sufficiently large conductor, being determined by molecular interactions. The concept of "ideal conductor" is not very restrictive, unless for a much-rarefied gas.

\subsection{Diffusion and convection}

General relations between "mass-energy" and "diffusion-convection-heat" systems of coordinates were discussed in Section 2.2.3 for the Knudsen gas. This discussion was rather general and remains true for dense mixtures. In particular, eqs. (34)-(39) for the Onsager coefficients in binary mixtures remain generally valid. The only equation that needs modification is the equation for the heat conductivity coefficient:

$$
L_{D, 22}=-\sum_{k=1}^{2}\left[h \lambda_{k}\left(h \frac{\partial \Gamma_{k}}{\partial v_{k}}-\frac{\partial \Gamma_{k}}{\partial \tau}\right)+\lambda_{k} \frac{\partial \Gamma_{k} G_{k}}{\partial \tau}+\lambda_{k}\left(g_{k}-h\right) \frac{\partial \Gamma_{k}}{\partial \tau}\right]-\lambda_{E} \frac{\partial \mathrm{E}_{n c}}{\partial \tau} .
$$

Since the expressions for the transport coefficients remain unchanged, the problem of coupling between diffusion/heat conduction and convection persists also for this case. Moreover, an explanation given in Section 2.2.3 does not work anymore, since it is specifically oriented onto a gas of non-interacting molecules. The cases of dense gas or liquid require a different analysis, referring to fundamentals of non-equilibrium thermodynamics.

At the core of the Onsager derivation of the symmetry property for the phenomenological transport coefficients lies an assumption that the directed flows and the flows arising from fluctuations of the thermodynamic values around equilibrium are equivalent. The phenomenological coefficients derived from the correlations between the fluctuations of the different values may as well be applied to describe the directed flows with well-established pressure, concentration, and temperature gradients [56, 57].

This assumption is validated when small deviations from equilibrium are considered since for such cases there seems to be no clear boundary or difference between the fluctuations and the directed flow. For the convective flows, however, such a difference does exist. Consider for example the case where the conductor is a straight cylindrical capillary. The directed viscous flow of a dense fluid in such a conductor obeys the Poiseuille law, with a parabolic velocity profile and the no-slip condition on the wall. Meanwhile, the fluctuations are more chaotic, and the molecular velocities attributed to the convective flow may be nearly the same close to the walls and away from them. While the fluctuating flow is close to uniform across the cross-section of the capillary, the directed flow, most likely, is not.

Decoupling of the viscous and diffusive flows in discontinuous systems is also largely based on the fact that the diffusion and heat conduction is essentially structure-less, while the viscous flow possesses a certain structure. This difference is linked to the difference between viscous forces and diffusion for the continuous systems, where the corresponding laws have different tensor dimensions.

For small deviations from equilibrium and random fluctuations in discontinuous systems, however, there may be not enough time to form the viscous flow structure until the pressure gradient will change. Diffusive behavior of molecules in fluctuations is the same as in the directed flows, while the convective flux may also be randomly fluctuating, not differently from the diffusion fluxes. This results in the coupling between the two kinds of fluxes. 
Thus, coupling between diffusion and convection, as well as heat flow and convection, is a phenomenon occurring in hypothetical discontinuous systems for small and random deviations from equilibrium. This coupling disappears when large-scale directed motion in such systems is considered. For binary systems, the coefficients $L_{D, 31}, L_{D, 13}, L_{D, 32}, L_{D, 23}$ become zero in a large-scale approximation, while the convective resistance coefficient $L_{D, 33}$ changes. The terms proportional to $\Delta P$ disappear in the expressions for diffusion and heat fluxes. Other terms and coefficients (diffusion, Soret, Dufour, heat conductivity) remain unchanged and may be used for both random fluctuations and large-scale directed flow, as well as for both discontinuous and continuous systems. Similar statements are valid for the general multicomponent systems.

\subsection{Conclusion}

We have developed a theory of transport properties, like diffusion and thermodiffusion coefficients, as well as heat conductivities, based on detailed consideration of the flows in non-equilibrium discontinuous systems. The theory may not be applied to computation of viscosities, and, hence, is not universal. However, it covers a wide range of practically important applications.

Our approach is based on explicitly formulated and physically meaningful assumptions, and generalizing the case of Knudsen flow onto arbitrary multicomponent gases and liquids. This generalization required a different energy flow equation and introduction of the arbitrary emission functions and penetration probabilities (or penetration lengths). Application of the Onsager symmetry property makes it possible to reduce the number of the necessary unknown functions and strongly restrict their dependencies on thermodynamic variables. As a result, a problem of determination of $(n+1)^{2}$ transport coefficients (or $n(n+1) / 2$, if symmetry is accounted for) is reduced to $2(n+1)$ emission functions and penetration lengths. All the emission functions $\Gamma_{i}$ depend only on temperature and a single reduced chemical potential $v_{i}$, while the non-convective heat emission $E_{n c}$ depends only on temperature $\tau$. The penetration lengths may in principle be determined in molecular dynamics simulation studies [53].

The properties of the transport coefficients are derived based on the Onsager symmetry (which, for this case, turns out to be rather restrictive) and on the independence of the properties of the conductor and vessels forming a discontinuous system. We have discussed a major theoretical difficulty: coupling between diffusive/heat and convective flows, which is present in the fluctuating discontinuous, but not in the continuous, systems, although some new formalisms suggest a possibility for such coupling.

\section{Examples}

\subsection{Ideal emission functions}

In this section, we will present examples of the transport coefficients calculated on the basis of the developed theory. The first step is to define the mass emission functions $\Gamma_{i}$. It may be tempting to postulate that they are equal to $\frac{1}{4} \rho_{i} C_{i}$, as for the ideal gas case (and as applied in [51, 52]). However, such a form is inconsistent with Statement 1 , since for non-ideal mixtures the values of $\rho_{i}$ may not only be dependent on $v_{i}$, but also on other potentials $v_{j}$.

According to eqs. (19) and (61), the values of $\Gamma_{i}$ for gas mixtures of non-interacting molecules have the form of

$$
\begin{gathered}
\Gamma_{i}=a_{i}(\tau) \exp \left(-\frac{M_{i}}{R} v_{i}\right), \\
a_{i}=A_{i}^{\prime} \tau^{\frac{c_{V, i}}{R}+\frac{1}{2}} .
\end{gathered}
$$

We will apply eq. (52) [with arbitrary $a_{i}$ or with $a_{i}$ in the form of eq. (53)] as a model expression for mass emission functions. Its thermodynamic meaning becomes evident if it is represented in the equivalent form

$$
\Gamma_{i}=a_{i}^{0}(T) f_{i}
$$


Here $f_{i}$ are fugacities and multipliers $b_{i}$ are combinations of the molecular velocities and chemical potentials in the reference state. Thus, the ideal model assumes that emissions are proportional to the component fugacities. This is in correspondence with the equilibrium condition of fugacity equality in the two vessels.

Consider now dense liquids or gases defined by eq. (51). The form (52) of the emission functions imposes the restrictions on the values $g_{i}$ of the energy transferred with the molecules.

Statement 4. If $\Gamma_{i}$ are defined as in eq. (52) (with arbitrary $a_{i}(\tau)$ ), then $g_{i}$ depend only on the temperature. Vice versa, if $g_{i}$ are only dependent on $\tau$, then $\Gamma_{i}$ have the form of

$$
\Gamma_{i}=\Phi_{i}\left\{a_{i}(\tau) \exp \left(-\frac{M_{i}}{R} v_{i}\right)\right\}
$$

where $\Phi_{i}$ are arbitrary functions.

Proof of the statement, as well as the relation between $g_{i}(\tau)$ and $a_{i}(\tau)$, is described in Appendix E.

In the following, we will use only the values of $\Gamma_{i}$ in the form (52), but not in a more general form presented in Statement 4, which needs a separate study.

\subsection{Diffusion coefficients}

\subsubsection{General formula}

In this section, we analyze expressions for diffusion coefficients in binary mixtures. The Onsager phenomenological coefficient for the diffusion of the Knudsen gas $L_{D, 11}$ is given by eq. (34), and this expression may be used without changes for an arbitrary binary mixture. A relationship between the Onsager and diffusion coefficients comes from the definition of the binary diffusive flux. Under constant pressure and temperature,

$$
J_{D}=L_{D, 11} \frac{\partial\left(v_{1}-v_{2}\right)}{\partial x}=-\rho D \frac{\partial c_{1}}{\partial x} .
$$

Hence, the binary (Fickian) diffusion coefficient is expressed by

$$
D=-\rho^{-1} L_{D, 11} \frac{\partial\left(v_{1}-v_{2}\right)}{\partial c_{1}} .
$$

The reduced chemical potentials $v_{i}$ obey the Gibbs-Duhem relation:

$$
c_{1} d v_{1}+c_{2} d v_{2}=0(P, T=\text { const }) .
$$

Hence, the last equation for the diffusion coefficient may be transformed to the form of (see Appendix B.2)

$$
D=-\rho^{-1} L_{D, 11} \frac{1}{c_{2}} \frac{\partial v_{1}}{\partial c_{1}}=\rho^{-1} L_{D, 11} \frac{R}{c_{1} c_{2} M_{1} M_{2}\left(\frac{c_{1}}{M_{1}}+\frac{c_{2}}{M_{2}}\right)}\left[1+M_{2} c_{1}\left(\frac{c_{1}}{M_{1}}+\frac{c_{2}}{M_{2}}\right) \frac{\partial \ln \gamma_{1}}{\partial c_{1}}\right]
$$

Here $\gamma_{1}$ is the activity coefficient. It may be shown that a cumbersome expression $M_{2} c_{1}\left(\frac{c_{1}}{M_{1}}+\frac{c_{2}}{M_{2}}\right) \frac{\partial \ln \gamma_{1}}{\partial c_{1}}$ is reduced to a compact form of $z_{1} \frac{\partial \ln \gamma_{1}}{\partial z_{1}}, z_{1}$ being the molar fraction of the first component, so that the expression in square parentheses is a usual thermodynamic correction [10]. The remaining part of the expression may be attributed as the ideal diffusion coefficient in the mass-related system of variables:

$$
D_{0}=\rho^{-1} L_{D, 11} \frac{R}{c_{1} c_{2} M_{1} M_{2}\left(\frac{c_{1}}{M_{1}}+\frac{c_{2}}{M_{2}}\right)} .
$$

It may sometimes be more convenient not to consider the flux proportionality to the mass fraction difference $\Delta c_{1}$, but to the difference of the molar fraction $\Delta z_{1}$. The diffusion flux may correspondingly be represented in the form

$$
J_{D}=-\rho D_{M S}\left(1+z_{1} \frac{\partial \ln \gamma_{1}}{\partial z_{1}}\right) \frac{\Delta z_{1}}{l}
$$


Here $D_{M S}$ is the so-called Maxwell-Stefan diffusion coefficient $[14,63,64]$, expressed by

$$
D_{M S}=\rho^{-1} L_{D, 11} \frac{R\left(\frac{c_{1}}{M_{1}}+\frac{c_{2}}{M_{2}}\right)}{c_{1} c_{2}} .
$$

An advantage of introducing coefficients $D_{0}, D_{M S}$ is that they show a simpler dependence on concentration than the coefficient $D$. In particular, the standard Darken and Vignes mixing rules $[6,7]$ may often be applied to predict their behavior over the whole range of concentrations [21, 63].

Finally, let us consider the case of ideal emission functions. The formulae of this section are applicable for this case, while the expression for $L_{D, 11}$ is simplified as follows:

$$
L_{D, 11}=\frac{M_{1}}{R} c_{2}^{2} \lambda_{1} \Gamma_{1}+\frac{M_{2}}{R} c_{1}^{2} \lambda_{2} \Gamma_{2} \text {. }
$$

\subsubsection{Ideal gas mixture}

The developed theory may be verified for the case of a binary mixture of ideal gases, where the diffusion coefficient is known from the Chapman-Enskog expansion of the kinetic Boltzmann equation [27]. The Boltzmann ideal gases may be denser than the Knudsen gas, since their molecules may participate in binary collisions. However, they still obey the ideal gas law. It may then be assumed that the emission functions $\Gamma_{i}$ are still equal to $\rho_{i} C_{i}$, as in eq. (19). Combination of eqs. (54) and (55), with the activity coefficient equal to unity, results in

$$
D=z_{2} \lambda_{1} C_{1}+z_{1} \lambda_{2} C_{2}
$$

Here $z_{i}$ are molar fractions.

Equation (56) is to be compared with the expression for the diffusion coefficient obtained in the framework of the gas kinetic theory:

$$
D=\frac{3 C_{12}}{16 N \sigma_{12}^{2}} ; C_{12}=\left(\frac{8 R T}{\pi M_{12}}\right)^{2} ; M_{12}=\left(\frac{1}{M_{1}}+\frac{1}{M_{2}}\right)^{-1} .
$$

This expression is valid if the penetration distances are equal to

$$
\lambda_{i}=\frac{3}{4} \frac{\left(\frac{M_{i}}{M_{12}}\right)^{\frac{1}{2}}}{\sigma_{12}^{2} N} .
$$

These results are worth comparing with the elementary molecular theory of diffusion [27]. This theory represents diffusion as a process of molecular exchange between adjacent molecular layers located at a distance of the free molecular path $\lambda_{f}$ from each other. The exchange frequency is determined by molecular velocities $C_{i}$, and average molecular migration occurs due to the disbalance of the molecular concentrations in the neighboring layers. Then eq. (56) for the diffusion coefficient may be recovered, with the values $\lambda_{f i}$ instead of $\lambda_{i}(i=1,2)$. Application of the well-known expressions for $\lambda_{f i}$ results in an expression that differs from the result (57) of the gas kinetic theory only by a numerical coefficient.

The values of $\lambda_{i}$, as determined by eq. (58), also differ from $\lambda_{f i}$ by a numerical coefficient. This provides a physical intuition behind eq. (56): Diffusion in ideal gases occurs by the molecular exchange between adjacent molecular layers located at a penetration distance from each other.

It should be noted that if $\lambda_{i}$ depend on $P, T$, but not on the mixture composition, then eq. (56) is equivalent to the Darken rule:

$$
D=z_{1} D\left(z_{1} \rightarrow 1\right)+z_{2} D\left(z_{2} \rightarrow 1\right) .
$$

However, this derivation of the Darken rule is of limited validity. For example, it presumes that the molar density is independent of the composition under constant pressure and temperature. A less restrictive derivation of the Darken or a similar mixing rule is presented in the next subsection. 


\subsubsection{Ideal mixture}

Let us now consider an ideal mixture, without the assumption that this is an ideal gas (that is, the ideal gas equation of state is not necessarily valid). The necessary thermodynamic formulae for this case are discussed in Appendix B.2. Assume that the emission functions are ideal, as in Section 4.1. Combining eqs. (52) and (68), we obtain

$$
\Gamma_{i}=b_{i}(P, \tau) z_{i} ; b_{i}=a_{i}(\tau) \exp \left(-\frac{M_{i}}{R} v_{i}^{0}\right) .
$$

The expressions for diffusion coefficients from Section 2.1 may be transformed into

$$
\begin{gathered}
L_{D, 11}=\frac{1}{R} \frac{c_{1} c_{2}}{\frac{c_{1}}{M_{1}}+\frac{c_{2}}{M_{2}}}\left(c_{2} \lambda_{1} b_{1}+c_{1} \lambda_{2} b_{2}\right) ; \\
D_{M S}=\rho^{-1}\left(c_{2} \lambda_{1} b_{1}+c_{1} \lambda_{2} b_{2}\right) ; \\
D=D_{0}=\rho^{-1}\left(c_{2} \lambda_{1} b_{1}+c_{1} \lambda_{2} b_{2}\right) \frac{1}{M_{1} M_{2}\left(\frac{c_{1}}{M_{1}}+\frac{c_{2}}{M_{2}}\right)^{2}} .
\end{gathered}
$$

Of these coefficients, $D_{M S}$ is expressed in the simplest way, and behaves the closest to the conventional Darken rule. This rule becomes valid in assumptions that the penetration distances $\lambda_{i}$ are dependent only on $T, P$ and that the density may be approximately taken to be constant. Then the linear mixing rule is valid with regard to the mass fractions, while $D_{M S}$ is the coefficient related to the case where the diffusion flux is expressed in terms of the molar fraction.

As shown by eq. (69), the density of an ideal liquid is a non-linear function of the mass fraction. Additionally, it has been verified in molecular dynamics simulations that the penetration distances may also depend on the mixture composition [53]. This is reflected in the weak non-linearity of the diffusion coefficients of nearly ideal mixtures [12]. On the other hand, the dependence of the penetration distances on the concentration in binary mixtures is usually relatively simple [14]. This is probably in correlation with the results of [65], where the matrix of the Onsager coefficients, at least for some systems, is demonstrated to factorize into a constant matrix and a scalar function of the mixture thermodynamics. This question needs further investigation.

\subsection{The thermodiffusion coefficient: Thermodynamic invariance}

Lastly, we present the equations for the thermodiffusion coefficient. This coefficient $D_{T}$ is defined as a coefficient at the temperature gradient in the expression for the diffusion flux $J_{D}$. Under constant pressure, this flux is expressed as

$$
J_{D}=-\rho\left(D \frac{\partial c_{1}}{\partial x}+D_{T} c_{1} c_{2} \frac{\partial T}{\partial x}\right) .
$$

This expression is to be compared with the Onsager relations (33) in the diffusion system of thermodynamic coordinates. Again, under constant pressure, the expression for the diffusion flux is

$$
J_{D}=L_{D, 11} \frac{\partial\left(v_{1}-v_{2}\right)}{\partial x}+L_{D, 12} \frac{\partial \tau}{\partial x} .
$$

Comparison of the two expressions results in

$$
D_{T}=\frac{T^{2}}{\rho c_{1} c_{2}} L_{D, 12}-\frac{1}{\rho c_{1} c_{2}} L_{D, 11} \frac{\partial\left(v_{1}-v_{2}\right)}{\partial T} .
$$

Here coefficients $L_{D, 11}$ and $L_{D, 12}$ are given by eqs. (34) and (37). In particular, for ideal emission functions, the coefficient $L_{D, 11}$ is given by eq. (55), while eq. (37) is transformed into

$$
L_{D, 12}=h\left(c_{1} \lambda_{2} \frac{M_{2}}{R} \Gamma_{2}-c_{2} \lambda_{1} \frac{M_{1}}{R} \Gamma_{1}\right)-c_{2} \lambda_{1} \frac{a_{1}^{\prime}}{a_{1}} \Gamma_{1}+c_{1} \lambda_{2} \frac{a_{2}^{\prime}}{a_{2}} \Gamma_{2} .
$$


An important checkpoint of expression (59) for the thermodiffusion coefficient is whether it is invariant with regard to changes in the reference states of the energy and entropy. In thermodynamics, these fundamental quantities are determined within a constant. Although the constant in the definition of the entropy is formally fixed by the Third Law of Thermodynamics (the Nernst theorem), in practice this determination is almost never applied, in view of the difficulty to build a continuous path of equilibrium transformation between a state with zero temperature and a given thermodynamic condition.

Consider the two different systems of thermodynamic variables ("with and without the star") corresponding to the two reference states. Assume that these reference states differ from each other by the energy and entropy constants $u_{0}, s_{0}$. Then

$$
\mu_{m, k}-\mu_{m, k}^{*}=u_{0}-T s_{0} ; v_{k}-v_{k}^{*}=s_{0}-u_{0} \tau .
$$

Obviously, the transport coefficients should remind invariant under such a transformation. However, this statement may not hold for some empirical or semi-empirical expressions for the thermodiffusion coefficients. Many models express the thermodiffusion coefficients in terms of the so-called heats of transport [30]. As an example, the expressions suggested by Haase [32] and Kempers [39, 40] express the thermodiffusion coefficients in terms of the partial molar enthalpies, which are not invariant with regard to the choice of the reference energy. See also the more detailed discussion in [66].

It may be checked, by cumbersome but routine computations, that all the transport coefficients, including the thermodiffusion coefficient (59), are invariant with regard to the change of the energy and entropy reference states.

\section{Conclusion}

In this work, we have demonstrated how the diffusion and thermodiffusion coefficients in multicomponent mixtures may be computed based on their thermodynamic properties. The derivation was based on a generalization of the properties of the Knudsen gas and followed the idea of the potential character of the flow in the discontinuous systems. Although the approach is not universal and cannot be easily generalized to the computation of viscosities, it covers a number of practically important cases. As a result, the set of $n(n+1) / 2$ independent Onsager coefficients was reduced to $2(n+1)$ dependencies: the emission functions and the penetration lengths. These functions have a clear physical significance. The Onsager symmetry property poses a number of strong limitations on the possible forms of these dependencies. Model expressions for them may be proposed. As shown in Section 4, relatively simple sets of penetration lengths and emission functions make it possible to match well-known expressions for diffusion coefficients in binary mixtures.

Acknowledgment: O. Medvedev, M. Bagnoli, F. Montel, G. Galliero, H. Wesselingh, and H. Baghooee are kindly acknowledged for multiple productive discussions.

Funding: This study was initiated in the framework of the Talent project granted by the Danish Technical Research Council, grant 25-00-0315.

\section{Appendix A. Kinetic derivation of the energy emission function for the Knudsen gas}

This appendix aims at explaining why the energy emission value has the form of eq. (9), including the term proportional to $R T / 2$.

Consider the energy emitted with the molecules from vessel $A$ into the conductor per unit of time:

$$
J_{A, E+}=H E_{A}=H N_{A} N_{A v}\left\langle v_{x} e\right\rangle_{+} .
$$


Here $N_{A v}$ is the Avogadro number, $N_{A}$ is the molar density in vessel $A, v_{x}$ is the $x$-component of the molecular velocity, and $e$ is the energy per molecule. Averaging \langle\rangle$_{+}$is taken over all the molecules for which $v_{x}>0$.

The overall energy flux may be found as

$$
J_{E}=J_{A, E+}-J_{B, E-}
$$

The value of $J_{B, E-}$ is symmetric to $J_{A, E+}$ and is calculated by the same rule.

The energy $e$ is a sum of the kinetic and internal energy of the molecule:

$$
e=\frac{m_{0}\left(v_{x}^{2}+v_{y}^{2}+v_{z}^{2}\right)}{2}+e_{i}
$$

In the average $\left\langle v_{x} e\right\rangle_{+}$, the multiplier $v_{x}$ is only correlated with the part $m_{0} v_{x}^{2} / 2$ of the expression for the energy. Therefore,

$$
\left\langle v_{x} e\right\rangle_{+}=\left\langle v_{x}\right\rangle_{+}\langle e\rangle+\frac{m_{0}}{2}\left(\left\langle v_{x}^{3}\right\rangle_{+}-\left\langle v_{x}\right\rangle_{+}\left\langle v_{x}^{2}\right\rangle\right) .
$$

The value of $\langle e\rangle$ in this expression is equal to $c_{V} T / N_{A v}$. Computation of the velocity averages is carried out over the Maxwell distribution $f_{M}\left(v_{x}\right)=\left(\frac{m_{0}}{2 \pi k T}\right)^{\frac{1}{2}} \exp \left(-\frac{m_{0} v_{x}^{2}}{2 k T}\right)$. The result is

$$
\begin{aligned}
\left\langle v_{x}\right\rangle_{+} & =\frac{1}{4} C ; \\
\left\langle v_{x}^{3}\right\rangle_{+}-\left\langle v_{x}\right\rangle_{+}\left\langle v_{x}^{2}\right\rangle & =\frac{1}{2} C \frac{k T}{2 m_{0}}=\frac{1}{2} C \frac{R T}{2 M} .
\end{aligned}
$$

Substitution of the last three equations back into eq. (60) results in

$$
\mathrm{E}_{A}=\frac{C}{4} \rho\left(c_{V}+\frac{R}{2}\right) T,
$$

as expected. It should be remarked that the addendum proportional $R T / 2$ comes directly from the average $\frac{m_{0}}{2}\left(\left\langle v_{x}^{3}\right\rangle_{+}-\left\langle v_{x}\right\rangle_{+}\left\langle v_{x}^{2}\right\rangle\right)$, that is, from the correlation between the velocity and the energy of the emitted molecules. Faster molecules have, on average, higher energies, which contributes to the energy flux.

\section{Appendix B. Useful thermodynamic expressions}

\section{B.1 General formulae}

This appendix brings together useful thermodynamic formulae, which are used in other sections. Derivation of most of them is straightforward but cumbersome.

Consider a vessel of a constant volume, like vessels $A$ and $B$ in the system described above. The differential of its entropy per unit volume, in variables $v_{i}, \tau$ has the form of

$$
d S=\sum_{i=1}^{n} v_{i} d \rho_{i}+\tau d u
$$

This shows that $v_{i}, \tau$ are derivatives of the entropy with regard to the extensive variables $\rho_{i}, u$, the last being the internal energy density.

The ideal-gas expressions for chemical potentials $\mu_{i}$ are well known [67]. The reduced chemical potentials $v_{i}$ are found to be

$$
v_{i}=-\frac{R}{M_{i}} \ln \rho_{i}-c_{V, i} \ln \tau+A_{0 i}
$$


Here and further in this section $A_{i}, A_{0 i}, \ldots$ are the different constants; the value of $\tau$ may be expressed in terms of the extensive variables as

$$
\tau=\frac{1}{u} \sum_{i=1}^{n} \frac{c_{V, i} \rho_{i}}{M_{i}}
$$

We will need inverse expressions for extensive variables $m_{i}, u$ in terms of $v_{i}, \tau$. Straightforward computations show that

$$
\begin{gathered}
\rho_{i}=A_{i} \tau^{\frac{c_{V, i}}{R}} \exp \left(-\frac{M_{i}}{R} v_{i}\right) ; \\
u=\sum_{i=1}^{n} \frac{c_{V, i} A_{i} \tau^{\frac{c_{V, i}}{R}-1} \exp \left(-\frac{M_{i}}{R} v_{i}\right)}{M_{i}} .
\end{gathered}
$$

The derivatives of these variables have the form of

$$
\begin{gathered}
\frac{\partial \rho_{i}}{\partial v_{j}}=-\delta_{i j} \frac{M_{i}}{R} A_{i} \tau^{\frac{c_{V, i}}{R}} \exp \left(-\frac{M_{i}}{R} v_{i}\right)=-\delta_{i j} \frac{M_{i}}{R} \rho_{i} ; \\
\frac{\partial \rho_{i}}{\partial \tau}=\frac{c_{V, i}}{R} A_{i} \tau^{\frac{c_{V, i}}{R}-1} \exp \left(-\frac{M_{i}}{R} v_{i}\right)=\frac{c_{V, i} \rho_{i}}{R} ; \\
\frac{\partial u}{\partial v_{i}}=-\frac{c_{V, i} A_{i} \tau^{\frac{c_{V, i}}{R}-1} \exp \left(-\frac{M_{i}}{R} v_{i}\right)}{R}=-\frac{c_{V, i} \rho_{i}}{R \tau} ; \\
\frac{\partial u}{\partial \tau}=\sum_{i=1}^{n} \frac{c_{V, i}\left(c_{V, i}-R\right) A_{i} \tau^{\frac{c_{V, i}}{R}-2} \exp \left(-\frac{M_{i}}{R} v_{i}\right)}{R M_{i}}=\sum_{i=1}^{n} \frac{c_{V, i}\left(c_{V, i}-R\right) \rho_{i}}{R \tau M_{i}} .
\end{gathered}
$$

Equations (62)-(65) form coefficients of the matrix $\left(d^{2} S\right)^{-1}$ inverse to the matrix of the second entropy derivatives with regard to its natural variables $\rho_{i}, u$.

\section{B.2 Mass vs molar}

In this paper, we prefer the system of thermodynamic variables related to mass densities and mass fractions. Meanwhile, most of the common equilibrium thermodynamic expressions (e. g., expressions for chemical potentials of ideal fluids) are formulated in terms of the molar quantities. It is worthwhile to provide a list of formulae governing the transformations from one system to another.

The relations between mass fractions $c_{i}$ and molar fractions $z_{i}$ are given by

$$
c_{i}=\frac{M_{i} z_{i}}{\sum_{j=1}^{n} M_{j} z_{j}} ; z_{i}=\frac{\frac{c_{i}}{M_{i}}}{\sum_{j=1}^{n} \frac{c_{j}}{M_{j}}} .
$$

For the binary mixture, the derivatives with regard to these variables (provided that $c_{2}$ and $z_{2}$ also vary with $c_{1}, z_{1}$ ) are related by

$$
\frac{\partial}{\partial z_{1}}=M_{1} M_{2}\left(\frac{c_{1}}{M_{1}}+\frac{c_{2}}{M_{2}}\right)^{2} \frac{\partial}{\partial c_{1}} ; \Delta c_{1}=M_{1} M_{2}\left(\frac{c_{1}}{M_{1}}+\frac{c_{2}}{M_{2}}\right)^{2} \Delta z_{1} .
$$

Consider now the chemical potential of the first component in an arbitrary binary mixture:

$$
v_{1}=v_{1}^{0}(P, T)-\frac{R}{M_{1}} \ln \left(z_{1} \gamma_{1}\right)=v_{1}^{0}(P, T)-\frac{R}{M_{1}} \ln \frac{c_{1}}{M_{1}}+\frac{R}{M_{1}} \ln \left(\frac{c_{1}}{M_{1}}+\frac{c_{2}}{M_{2}}\right)-\frac{R}{M_{1}} \ln \gamma_{1} .
$$

Differentiation by $c_{1}$ under constant $P, T$, taking into account that $c_{2}=1-c_{1}$, results in

$$
\frac{\partial v_{1}}{\partial c_{1}}=-\frac{R}{M_{1} M_{2}} \frac{1}{c_{1}\left(\frac{c_{1}}{M_{1}}+\frac{c_{2}}{M_{2}}\right)}-\frac{R}{M_{1}} \frac{\partial y_{1}}{\partial c_{1}} .
$$


This is the result used in Section 4.2.

Now we return to eq. (67) and consider it for the case of ideal mixture, $\gamma_{1}=1$. The value $z_{1}$ is expressed in terms of the chemical potentials, and the same is valid for $z_{2}$ :

$$
z_{i}=\exp \left(\frac{M_{i}}{R}\left(v_{i}^{0}-v_{i}\right)\right)
$$

Combination with eq. (66) gives

$$
c_{i}=\frac{M_{i} \exp \left(\frac{M_{i}}{R}\left(v_{i}^{0}-v_{i}\right)\right)}{M_{1} \exp \left(\frac{M_{1}}{R}\left(v_{1}^{0}-v_{1}\right)\right)+M_{2} \exp \left(\frac{M_{2}}{R}\left(v_{2}^{0}-v_{2}\right)\right)} .
$$

Further, for an ideal liquid the molar volumes $V_{i}$ are equal to individual molar volumes $V_{i}^{0}(P, T)$, which is easily verified by differentiation of eq. (67) by pressure. The total molar volume is equal to

$$
V=z_{1} V_{1}^{0}+z_{2} V_{2}^{0}
$$

From this equation, with application of eq. (66), it may be derived that

$$
\rho=\frac{1}{\frac{c_{1}}{\rho_{1}^{0}}+\frac{c_{2}}{\rho_{2}^{0}}}, \rho_{0}^{i}=\frac{M_{i}}{V_{i}^{0}} .
$$

\section{Appendix C. Different systems of thermodynamic forces and fluxes}

The goal of this appendix is to establish the relations between mass-energy and diffusion-heat-convection systems of thermodynamic forces and fluxes. The expressions for diffusive $J_{D, i}$, convective $J_{c}$, and heat flux $J_{Q}$, in terms of mass and energy fluxes $J_{m, i}, J_{E}$ are specified in eq. (31). The vectors of fluxes $\boldsymbol{J}_{M}=\left(J_{m, 1}, \ldots, J_{m, n}, J_{E}\right)^{T}$ and $\boldsymbol{J}_{D}=\left(J_{D, 1}, \ldots, J_{D, n-1}, J_{Q}, J_{C}\right)^{T}$ are conjugate to the corresponding vectors of thermodynamic forces $\boldsymbol{F}_{M}, \boldsymbol{F}_{D}$ :

$$
\frac{d S}{d t}=H \boldsymbol{J}_{M}^{T} \boldsymbol{F}_{M}=H \boldsymbol{J}_{D}^{T} \boldsymbol{F}_{D}
$$

The vector $\boldsymbol{F}_{M}$ is equal to $\left(\Delta v_{1}, \ldots, \Delta v_{M}, \Delta \tau\right)^{T}$. The goal is to calculate vector $\boldsymbol{F}_{D}$.

Since $\boldsymbol{J}_{M}$ and $\boldsymbol{J}_{D}$ are connected by linear relations, a formal calculation would be possible by application of straightforward linear algebra. However, such a computation is rather cumbersome. We will show a less straightforward, but a more elegant way.

Consider the differential of entropy, represented in terms of mass quantities:

$$
\Delta S=\tau \Delta U+P \tau \Delta V+\sum_{i=1}^{n} v_{i} \Delta m_{i}
$$

A Gibbs-Duhem relation for such a form of the basic thermodynamic equation is

$$
U \Delta \tau+V \Delta(P \tau)+\sum m_{i} \Delta v_{i}=0
$$

or, dividing by the total mass $m$,

$$
u \Delta \tau+\frac{1}{\rho} \Delta(P \tau)+\sum c_{i} \Delta v_{i}=0
$$

Decomposition of the product $\Delta(P \tau)$ gives

$$
h \Delta \tau+\frac{\tau}{\rho} \Delta P+\sum c_{i} \Delta v_{i}=0 .
$$


Here enthalpy per unit mass $h$ is equal to $u+P / \rho$.

Consider now the entropy production in the first form (70):

$$
\frac{d S}{d t}=\sum_{i=1}^{n} J_{m, i} \Delta v_{i}+J_{E} \Delta \tau
$$

Expressing $J_{m, i}$ as $J_{D, i}+c_{i} J_{c}$ and applying the Gibbs-Duhem relation in the form of (71), we obtain

$$
\frac{d S}{d t}=\sum_{i=1}^{n} J_{D, i} \Delta v_{i}-\left(h \Delta \tau+\frac{\tau}{\rho} \Delta P\right) J_{c}+J_{e} \Delta \tau
$$

A final transformation is obtained by noting that

$$
\begin{gathered}
J_{e}-h J_{c}=J_{Q} ; \\
\sum_{i=1}^{n} J_{D, i} \Delta v_{i}=\sum_{i=1}^{n-1} J_{D, i} \Delta\left(v_{i}-v_{n}\right) .
\end{gathered}
$$

The last equality is valid, since the sum of diffusion fluxes is zero. Substituting the last two equalities into eq. (72), we obtain

$$
\frac{d S}{d t}=\sum_{i=1}^{n-1} J_{D, i} \Delta\left(v_{i}-v_{n}\right)+J_{Q} \Delta \tau-J_{c} \frac{\tau}{\rho} \Delta P .
$$

The last equality proves that the vector $\boldsymbol{F}_{D}$ of thermodynamic forces conjugate to $\boldsymbol{J}_{D}$ is really given by eq. (32).

\section{Appendix D. Stochastic substantiation for the relationships between penetration probabilities and penetration distances}

In this appendix, we provide the stochastic arguments proving that a probability $p_{i}$ for a molecule to pass the conductor from vessel $A$ to vessel $B$ is equal to

$$
p_{i}=\frac{\lambda_{i}}{l}
$$

Here $\lambda_{i}$ is a penetration distance defined as such a distance, after which the molecule "forgets" its initial velocity and starts unbiased random walks.

The motion of a molecule in gas or liquid is a random process, which, depending on the fluid density, may either be represented as a discrete random walk or as a process with continuous trajectories. If a molecule forgets its initial state, this process is unbiased: Displacements of a molecule towards $A$ are equiprobable with displacements towards $B$. The unbiased walk starts when a molecule reaches a distance $\lambda_{i}$ from vessel $A$.

Let us first discuss a discrete random-walk case and consider the simplest model problem of random walks "by tossing a coin" (as discussed in [68, Chapter 3]). Assume that at each step the particle moves on +1 or -1 with a probability of $1 / 2$. It starts motion from position $I$ (corresponding to the penetration distance). What will be the probability that the molecule reaches position $N$ (vessel $B$ ) before returning to zero (vessel $A$ )?

A solution to this problem is as follows. Let $a_{I}$ be a probability to reach $N$ before zero. After the first step of the random walk, the particle moves to positions $I+1$ or $I-1$ with equal probability $1 / 2$. The probability of final achievement of the position $N$ starting from $I$ is just an average of such probabilities from positions $I+1$ and $I-1$ :

$$
a_{I}=\frac{1}{2}\left(a_{I+1}+a_{I-1}\right), \text { or } a_{I+1}-a_{I}=a_{I}-a_{I-1}
$$


Thus, numbers $a_{I}$ form an arithmetic progression. Additionally, $a_{0}=0$ and $a_{N}=1$. So, the only possible solution is $A_{I}=\frac{I}{N}$, as required.

Consider now an opposite case of a particle motion in liquid, with a random continuous trajectory. The consideration follows [69, Chapter 10]. Let $q_{t}(x, Y)$ be a transition probability from point $x$ to a set $Y$ during time $t$. Such a probability obeys the backward Kolmogorov equation

$$
\frac{\partial q_{t}(x, Y)}{\partial t}=b \frac{\partial q_{t}(x, Y)}{\partial x^{2}} .
$$

Consider now a probability $Q(x)$ of achieving position $l$ (vessel $B$ ) before 0 (vessel $A$ ). For a reasonably small $t$, the integral backward equation has the form of [analogous to eq. (73)]

$$
Q(x)=\int q_{t}(x, d y) Q(y)
$$

Double-differentiating by $x$ and applying eq. (74), we obtain

$$
a \frac{\partial^{2} Q}{\partial x^{2}}=\int \frac{\partial q_{t}(x, d y)}{\partial t} Q(y) .
$$

Now, the last equality is integrated between arbitrary times $t_{1}$ and $t_{2}$, utilizing the fact that the left-hand side of the last equation is independent of $t$ :

$$
a\left(t_{2}-t_{1}\right) \frac{\partial^{2} Q}{\partial x^{2}}=\int\left[q_{t_{2}}(x, d y)-q_{t_{1}}(x, d y)\right] Q(y)
$$

Comparison with eq. (75) results in

$$
a\left(t_{2}-t_{1}\right) \frac{\partial^{2} Q}{\partial x^{2}}=Q(x)-Q(x)=0
$$

Thus, $\frac{\partial^{2} Q}{\partial x^{2}}=0$, or $Q$ is a linear function of $x$ equal to zero at $x=0$ and unity at $x=l$. The only solution for $Q(x)$ is $x / l$, as required.

Finally, let us consider the case where a particle performs random walks with a discrete step, whose length distribution is $q(\xi)$. This distribution is supposed to be an even function of $\xi$ (or, more generally, a distribution with a zero average). The equation for the probability $Q(x)$ for this case has the form of

$$
Q(x)=\int q(\xi) Q(x-\xi) d \xi
$$

A linear function $Q(x)$ is still a solution of eq. (76). However, there may formally be other solutions with fast oscillations within the characteristic step length. Additionally, the formal problems may arise if $q(\xi)$ has long "tales" extending outside the interval $[0, l]$. Detailed analysis and overcoming these difficulties is outside of the scope of the present work.

\section{Appendix E. Proof of Statement 4}

In this appendix we prove Statement 4 stating that in a dense mixture, under ideal emission functions given by eq. (52), the energy $g_{i}$ transferred with a molecule depends only on the temperature $T$; and a statement inverse to it. A basis for the proof is eq. (51), which, depending on a goal, may either be applied as an expression for $g_{i}$ in terms of $\Gamma_{i}$, or as a hyperbolic equation for $\Gamma_{i}$ if $g_{i}$ is known.

The direct proof is straightforward. Rewrite eq. (51) in the form of

$$
g_{i}=-\frac{T^{2} \frac{\partial \Gamma_{i}}{\partial T}}{\frac{\partial \Gamma_{i}}{\partial v_{i}}}
$$


Substitution of $\Gamma_{i}$ from eq. (52) results in

$$
g_{i}=\frac{R T^{2}}{M_{i}} \frac{a_{i}^{\prime}}{a_{i}}
$$

Since the values $a_{i}$ depend only on temperature, so do $g_{i}$.

Equation (77) makes it also possible to express $a_{i}$ in terms of $g_{i}$. This is the first-order differential equation that may be solved by separation of variables. The solution is

$$
a_{i}=\exp \left(\int \frac{M g_{i}}{R T^{2}} d T\right)
$$

Inversely, assume that values $g_{i}$ depend only on the temperature. Then it is possible to express them in the form (77) [simply, apply $a_{i}$ from eq. (78)]. Equation (51) is then reduced to the form

$$
\frac{\partial \Gamma_{i}}{\partial T}+\frac{R}{M_{i}} \frac{a_{i}^{\prime}}{a_{i}} \frac{\partial \Gamma_{i}}{\partial v_{i}}=0
$$

This is a hyperbolic equation for $\Gamma_{i}\left(v_{i}, T\right)$. Its general solution is

$$
\Gamma_{i}=\Phi_{i}^{\prime}\left(\ln a_{i}-\frac{M_{i}}{R} v_{i}\right),
$$

or, equivalently,

$$
\Gamma_{i}=\Phi_{i}\left(a_{i} \exp \left(-\frac{M_{i}}{R} v_{i}\right)\right) .
$$

This finishes the proof of the statement.

\section{List of designations}

a Pre-exponential factor in the ideal emission function

$b \quad$ Multiplier in the expression for the ideal emission function

c Mass fraction

$c_{V} \quad$ Heat capacity at constant volume

$c_{P} \quad$ Heat capacity at constant pressure

C Molecular velocity

$D_{X} \quad$ Energy per unit mass of emitted molecules

$D \quad$ Diffusion coefficient

$D_{T} \quad$ Thermodiffusion coefficient

$e \quad$ Energy of a single molecule

$f \quad$ Fugacity

$F \quad$ Thermodynamic force

$g \quad$ Part of the emitted energy lost in exchange with other molecules

$G \quad$ Preserved part of the emitted energy

$h \quad$ Enthalpy per unit mass

$H$ Cross-section of the conductor

$J \quad$ Flux

$k \quad$ Boltzmann constant

$\boldsymbol{K}$ Transformation matrix between the different systems of thermodynamic variables

$l \quad$ Length of the conductor

$L \quad$ Onsager phenomenological coefficient

L Matrix of the Onsager coefficients 


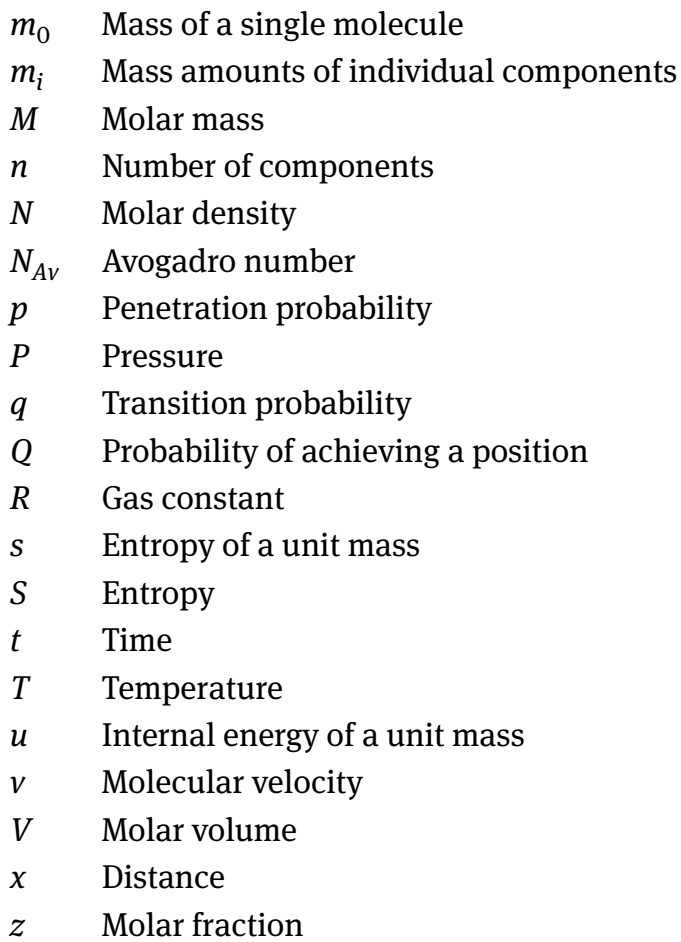

\section{Greek}

$y \quad$ Activity

$\Gamma \quad$ Mass emission function

E Energy emission function

$\Phi$ Functional dependence

$\lambda \quad$ Penetration length

$\mu \quad$ Chemical potential per unit mass of a component

$v \quad$ Reduced chemical potential

$\rho \quad$ Density

$\sigma \quad$ Collision diameter

$\tau \quad$ Inverse temperature

\section{Subscripts}

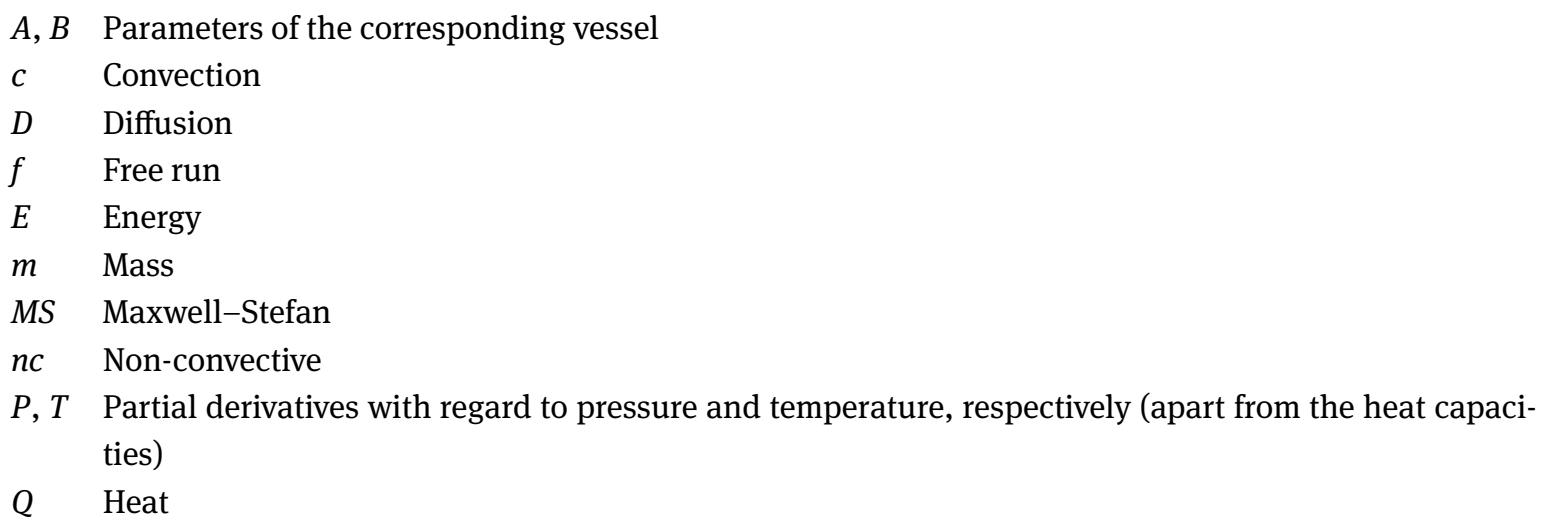




\section{References}

[1] C. Peters, J. Thien, L. Wolff, H. J. Koß and A. Bardow, Quaternary Diffusion Coefficients in Liquids from Microfluidics and Raman Microspectroscopy: Cyclohexane + Toluene + Acetone + Methanol, J. Chem. Eng. Data 65 (2020), no. 3, $1273-1288$.

[2] C. Peters, L. Wolff, S. Haase, J. Thien, Th. Brands and H. J. Koß, Multicomponent diffusion coefficients from microfluidics using Raman microspectroscopy, Lab Chip 17 (2017), no. 16, 2768-2776.

[3] E. D. Chisholm and D. C. Wallace, Dynamics of monatomic liquids: Topical review, J. Phys. Condens. Matter 13 (2001), no. 37, R739-R769.

[4] X. Liu, S. K. Schnell, J. -M. Simon, P. Krüger, D. Bedeaux, S. Kjelstrup, et al., Diffusion coefficients from molecular dynamics simulations in binary and ternary mixtures, Int. J. Thermophys. 34 (2013), 1169-1196.

[5] G. Galliero, M. Bugel, B. Duguay and F. Montel, Mass effect on thermodiffusion using molecular dynamics, J. Non-Equilib. Thermodyn. 32 (2007), no. 3, 251-258.

[6] L. S. Darken, Diffusion, mobility, and their interrelation through free energy in binary metallic systems, Trans. AIME, 175 (1948), 184-201.

[7] A. Vignes, Diffusion in binary solutions - variation of diffusion coefficient with composition, Ind. Eng. Chem. Fundam. 5 (1966), no. 2, 189-199.

[8] G. D. Moggeridge, Prediction of the mutual diffusivity in binary non-ideal liquid mixtures from the tracer diffusion coefficients, Chem. Eng. Sci. 71 (2012), 226-238.

[9] G. D. Moggeridge, Prediction of the mutual diffusivity in binary liquid mixtures containing one dimerizing species, from the tracer diffusion coefficients, Chem. Eng. Sci. 76 (2012), 199-205.

[10] E. K. Cussler, Diffusion: Mass transfer in fluid systems, Cambridge University Press, 1997.

[11] J. C. Bosma and J. A. Wesselingh, Estimation of diffusion coefficients in dilute liquid mixtures, Chem. Eng. Res. Des. 77 (1999), no. A6, 325-328.

[12] 0. O. Medvedev and A. A. Shapiro, Modeling diffusion coefficients in binary mixtures, Fluid Phase Equilib. 225 (2004), $13-22$.

[13] 0. O. Medvedev and A. A. Shapiro, Modeling diffusion coefficients in binary mixtures of polar and non-polar compounds, Fluid Phase Equilib. 236 (2005), 111-124.

[14] O. O. Medvedev, Diffusion Coefficients in Multicomponent Mixtures, Ph.D. Thesis, DTU, Kgs. Lyngby, Denmark, 2004.

[15] Y. -D. Hsu and Y. -P. Chen, Correlation of the mutual diffusion coefficients of binary liquid mixtures, Fluid Phase Equilib. 152 (1998), 149-168.

[16] G. D. Moggeridge, A local composition model for prediction of mutual diffusion coefficients in binary liquid mixtures from the tracer diffusion coefficients, Chem. Eng. Sci. 132 (2015), 250-258.

[17] M. Zhou, X. Yuan, Y. Zhang and K. T. Yu, Local composition based Maxwell-Stefan diffusivity model for binary liquid systems, Ind. Eng. Chem. Res. 52 (2013), 10845-10852.

[18] J. Li, H. Liu and Y. Hu, A mutual-diffusion-coefficient model based on local composition, Fluid Phase Equilib. 187-188 (2001), 193-208.

[19] D. Bosse and H. -J. Bart, Prediction of diffusion coefficients in liquid systems, Ind. Eng. Chem. Res. 45 (2006), $1822-1828$.

[20] D. Bosse and H. -J. Bart, Diffusion in associating liquid systems, Chem. Eng. Technol. 26 (2003), 1184-1188.

[21] J. A. Wesselingh and A. M. Bollen, Multicomponent diffusivities from the free volume theory, Chem. Eng. Res. Des. 75A (1997), 590-602.

[22] H. Liu, C. M. Silva and E. A. Macedo, Generalised free-volume theory for transport properties and new trends about the relationship between free volume and equations of state, Fluid Phase Equilib. 202 (2002), no. 1, 89-107.

[23] J. H. Dymond and M. A. Awan, Correlation of high-pressure diffusion and viscosity coefficients for n-alkanes, Int. J. Thermophys. 10 (1989), no. 5, 941-951.

[24] R. Qian, F. Yiqun, S. Meiren and S. Jun, Predictive equation of tracer liquid diffusion coefficient from viscosity, Chin. J. Chem. Eng. 4 (1996), no. 3, 203-208.

[25] V. V. Obukhovsky, A. M. Kutsyk, V. V. Nikonova and 0. O. Ilchenko, Nonlinear diffusion in multicomponent liquid solutions, Phys. Rev. E 95 (2017), 022133.

[26] E. A. Mason and A. P. Malinauskas, Gas transport in porous media: The dusty gas model, Elsevier, Amsterdam/New York, 1983.

[27] J. O. Hirschfelder, C. F. Curtiss and R. B. Bird, The molecular theory of gases and liquids, John Wiley, New York, 1954.

[28] M. B. Romero and R. M. Velasco, Onsager's symmetry in the Burnett regime, Physica A 222 (1995), no. 1-4, 161-172.

[29] V. I. Roldugin, The Chapman-Enskog theory and non-equilibrium thermodynamics, J. Non-Equilib. Thermodyn. 9 (1984), no. 1, 71-80.

[30] K. G. Denbigh, Thermodynamics of the steady state, Methuen \& Co. LDT, London, 1951.

[31] K. G. Denbigh, The heat of transport in binary regular solutions, Trans. Faraday Soc. 48 (1952), no. 1, 1-8.

[32] R. Haase, Thermodynamics of Irreversible Processes, Addison Welsey, London, 1969.

[33] W. M. Rutherford and H. G. Drickamer, Theory of thermal diffusion in liquids and the use of pressure to investigate the theory, J. Chem. Phys. 22 (1954), no. 7, 1157-1165. 
[34] E. L. Dougherty and H. G. Drickamer, A theory of thermal diffusion in liquids, J. Chem. Phys. 23 (1955), no. 2, 295-309.

[35] E. L. Dougherty and H. G. Drickamer, Thermal diffusion and molecular motion in liquids, J. Chem. Phys. 59 (1955), 443-444.

[36] L. J. Tichacek, W. S. Kmak and H. G. Drickamer, Thermal diffusion in liquids: the effect of non-ideality ans association, J. Phys. Chem. 60 (1956), 660-665.

[37] J. Shieh, Thermal diffusion and segmental motion in binary n-alkane systems, J. Phys. Chem. 73 (1969), no. 5, $1508-1513$.

[38] K. Shukla and A. Firozaabadi, A new model of thermal diffusion coefficients in binary hydrocarbon mixtures, Ind. Eng. Chem. Res. 37 (1998), 3331-3342.

[39] L. J. T. M. Kempers, A thermodynamic theory of the Soret effect in a multicomponent liquid, J. Chem. Phys. 90 (1989), no. 11, 6541-6548.

[40] L. J. T. M. Kempers, A comprehensive thermodynamic theory of the Soret effect in a multicomponent gas, liquid or solid, J. Chem. Phys. 15 (2001), no. 11, 6330-6341.

[41] K. Ghorayeb and A. Firoozabadi, Molecular, pressure, and thermal diffusion in non-ideal multicomponent mixtures, AIChE J. 46 (2000), no. 5, 883-891.

[42] M. Bagnoli, Modeling the thermal diffusion coefficients, Ph.D. Thesis, DTU, Kgs. Lyngby, 2004.

[43] M. A. Rahman and Z. M. Saghir, Thermodiffusion or Soret effect: Historical review, Int. J. Heat Mass Transf. 73 (2014), 693-705.

[44] S. Seshasai and Z. M. Saghir, Thermodiffusion Models, in: Springerbriefs in Applied Science and Technology (2013), 11-55. ISBN 978-1-4614-5598-1.

[45] K. Shukla, Statistical thermodynamics of thermal diffusion factors in binary hydrocarbon mixtures - an application, Mol. Phys. 115 (2017), no. 9-12, 1253-1263.

[46] F. Montel, H. Hoang and G. Galliero, Linking up pressure, chemical potential and thermal gradients, Eur. Phys. J. E 42 (2019), 65.

[47] E. Lapeira, M. Gebhardt, T. Triller, A. Mialdun, W. Köhler, V. Shevtsova, et al., Transport properties of the binary mixtures of the three organic liquids toluene, methanol, and cyclohexane, J. Chem. Phys. 146 (2017), 094507.

[48] M. Braibanti, P. A. Artola, P. Baaske, H. Bataller, J. P. Bazile, M. M. Bou-Ali, et al., European Space Agency experiments on thermodiffusion of fluid mixtures in space, Eur. Phys. J. E 42 (2019), 86.

[49] S. R. De Groot and P. Mazur, Non-equilibrium thermodynamics, New Holland Publications, 1962.

[50] G. D. C. Kuiken, Thermodynamics of irreversible processes, John Wiley \& Sons, 1994.

[51] A. A. Shapiro, Evaluation of diffusion coefficients in multicomponent mixtures by means of the fluctuation theory, Physica A 320 (2003), 211-234.

[52] A. A. Shapiro, Fluctuation theory for transport for transport properties in multicomponent mixtures: Thermodiffusion and heat conductivity, Physica A 322 (2004), 151-175.

[53] G. Galliero, 0. O. Medvedev and A. A. Shapiro, Molecular dynamics simulations of the penetration lengths: application within the fluctuation theory for diffusion coefficients, Physica A 350 (2005), 315-317.

[54] L. Waldmann, Non-equilibrium thermodynamics of boundary conditions, Z. Naturforsch. (1967), 1269-1280.

[55] F. Sharipov and D. Kalempa, Gaseous mixture flow through a long tube at arbitrary Knudsen numbers, J. Vac. Sci. Technol. A 20 (2002), 814-822.

[56] L. Onsager, Reciprocal relations in irreversible processes, Phys. Rev. 37 (1931), 405-426.

[57] L. Onsager, Reciprocal relations in irreversible processes, Phys. Rev. 38 (1931), 2265-2279.

[58] R. Wang, X. Xu, K. Xu and T. Qian, Onsager's cross coupling effects in gas flows confined to microchannels, Phys. Rev. Fluids 1 (2016), 044102.

[59] G. A. Bird, Molecular gas dynamics, Oxford University Press, 1976.

[60] O. O. Medvedev and A. A. Shapiro, Verifying reciprocal relations for experimental diffusion coefficients in multicomponent mixtures, Fluid Phase Equilib. 208 (2003), no. 1-2, 291-301.

[61] A. A. Shapiro, P. K. Davis and J. L. Duda, Diffusion in multicomponent mixtures, in: R. Gani and G. Kontogeorgis (eds.), Computer Aided Property Estimation, Elsevier, Amsterdam (2004), 205-228.

[62] M. Pavelka, F. Maršik and V. Klika, Consistent theory of mixtures on different levels of description, Int. J. Eng. Sci. 78 (2014), 192-217.

[63] A. A. Shapiro and E. H. Stenby, Factorization of transport coefficients in macroporous media, Transp. Porous Media 41 (2000), no. 3, 305-323.

[64] J. A. Wesselingh and R. Krishna, Mass transfer in multicomponent mixtures, VSSD, 2000.

[65] V. Klika, M. Pavelka and J. B. Benziger, Functional constraints on phenomenological coefficients, Phys. Rev. E 95 (2017), 022125.

[66] E. Bringuier, Gauge-invariant approach to thermodiffusion in a liquid binary mixture, Physica A 390 (2011), 1861-1875.

[67] M. L. Michelsen, J. Mollerup, Thermodynamic models: Fundamentals and computational aspects, Tie-Line Publications, 2004.

[68] W. Feller, An Introduction to Probability Theory and Its Applications, 1, John Wiley \& Sons, 1968.

[69] W. Feller, An Introduction to Probability Theory and Its Applications, 2, John Wiley \& Sons, 1968. 